\title{
Supporting Information for Three-Dimensionally Homoconjugated Carbon-Bridged Oligophenylenevinylene for Perovskite Solar Cells
}

Qifan Yan, Yunlong Guo,* Anna Ichimura, Hayato Tsuji,* and Eiichi Nakamura*

\author{
Table of contents
}

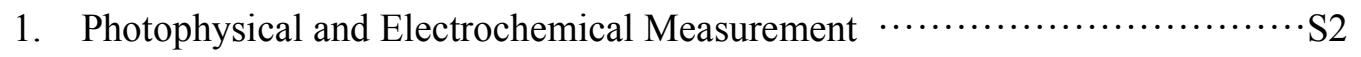

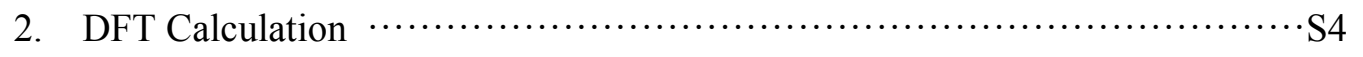

3. Energy levels, film XRD, UV-vis spectra, morphology analysis and J-V

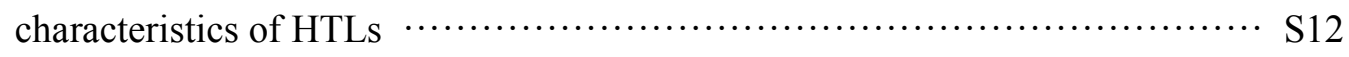

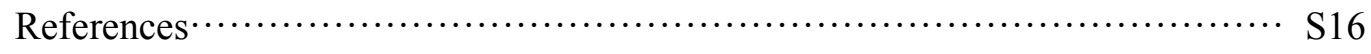

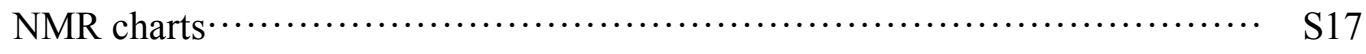




\section{Photophysical and Electro-chemical measurement}

Electro-chemical measurement was carried out in a standard one-compartment cell on a HZ-5000 voltammetric analyzer (HOKUTO DENKO). A common three-electrode configuration was adopted, using a Pt-wire counter electrode, a $\mathrm{Ag} / \mathrm{AgNO}_{3}$ reference electrode, and a glassy carbon working electrode. A sample was dissolved in $\mathrm{CH} 3 \mathrm{CN} / \mathrm{ODCB}(\mathrm{v} / \mathrm{v}, 1: 4)$ solution, with $\mathrm{Bu}_{4} \mathrm{NPF}_{6}$ at a concentration of $0.1 \mathrm{M}$ as supporting electrolyte. Oxidation of ferrocene $\left(\mathrm{Fc}^{+} / \mathrm{Fc}\right.$ couple) was used as external reference.

UV-vis-NIR absorption spectra were recorded on a JASCO-V670 spectrometer. Fluorescence emission spectra were recorded on a F-4500 fluorometer (HITACHI). Fluorescence quantum yield was measured with absolute method cyclic voltammetry on a C9920-02G spectrometer (Hamamatsu Photonics) equipped with integrating sphere. Titration experiment was done by adding a solution of $\mathrm{NO}^{+} \mathrm{PF}_{6}^{-}$in $\mathrm{CH}_{3} \mathrm{CN}$ into the UV-vis-NIR samples. 

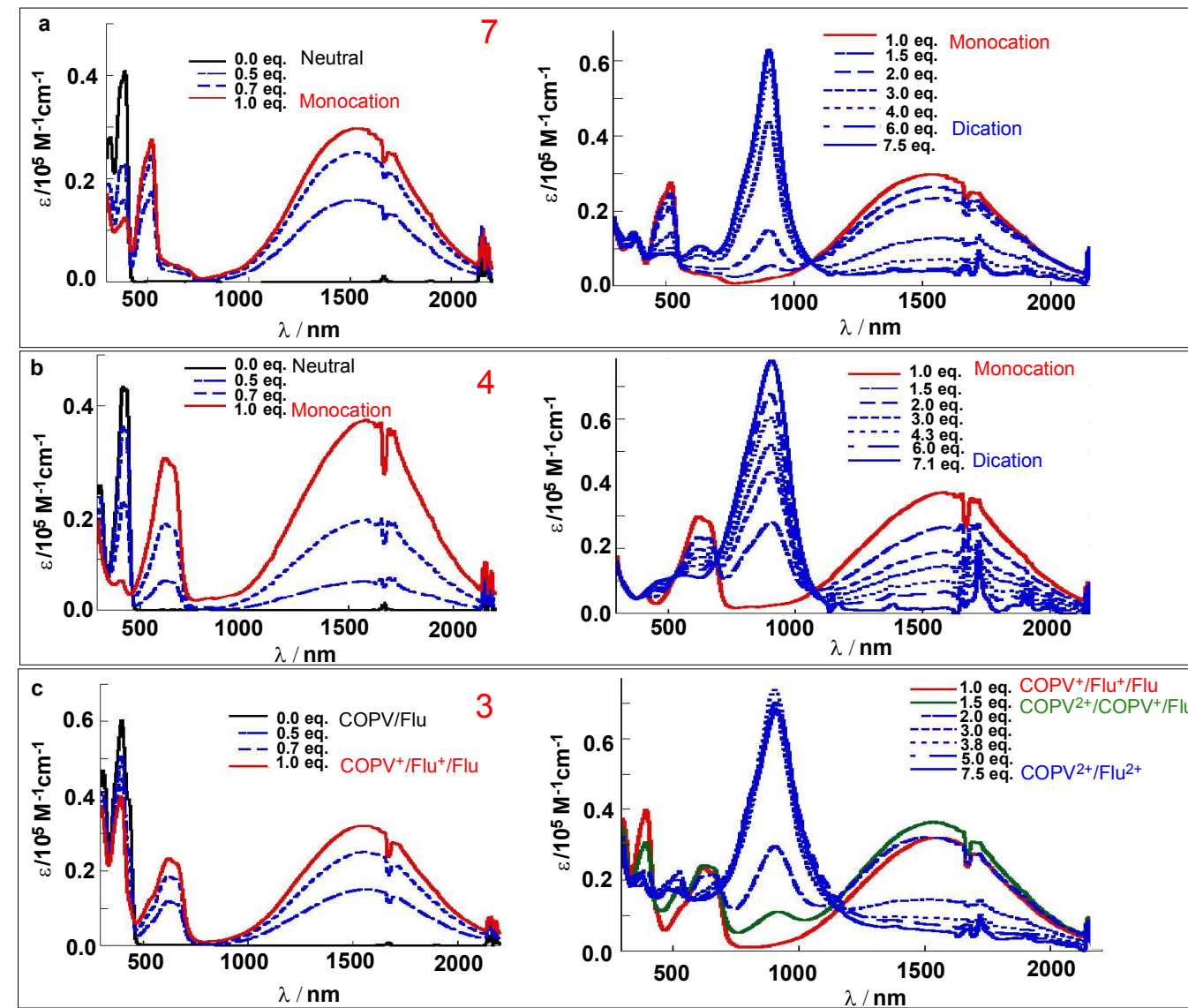

Figure S1. UV-vis-NIR absorption titration experiments. 


\section{DFT Calculations}

The geometry of neutral molecules and monocations was optimized with Density Functional Theory (DFT) using B3LYP hybrid functional ${ }^{\mathrm{S} 1}$ with a basis set limited to 6-31G(d,p). TD-DFT calculations were performed at optimized geometries using the same theory level. Quantum-chemical calculation was performed with the Gaussian 09, Revision B.01. ${ }^{\text {S2 }}$ All $n$-octyl substituents were replaced with methyl groups in calculations. Charge distribution of monocations was calculated by comparing between monocations and corresponding neutral molecules. Hole reorganization energy is calculated with a 4-point method as,

$$
\lambda_{\text {hole }}=\mathrm{E}^{+}(\mathrm{A})-\mathrm{E}^{+}\left(\mathrm{A}^{+}\right)+\mathrm{E}\left(\mathrm{A}^{+}\right)-\mathrm{E}(\mathrm{A})
$$

in which, $\lambda_{\text {hole }}$ is hole reorganization energy, $\mathrm{E}^{+}(\mathrm{A})$ is the energy of a cation calculated with the optimized structure of the neutral molecule, $\mathrm{E}^{+}\left(\mathrm{A}^{+}\right)$is the energy of a cation calculated with the optimized structure of the cation molecule, $\mathrm{E}\left(\mathrm{A}^{+}\right)$is the energy of a neutral molecule calculated with the optimized structure of the cation, E(A) is the energy of a neutral molecule calculated with the optimized structure of the neutral molecule. $^{\mathrm{S} 3}$ 

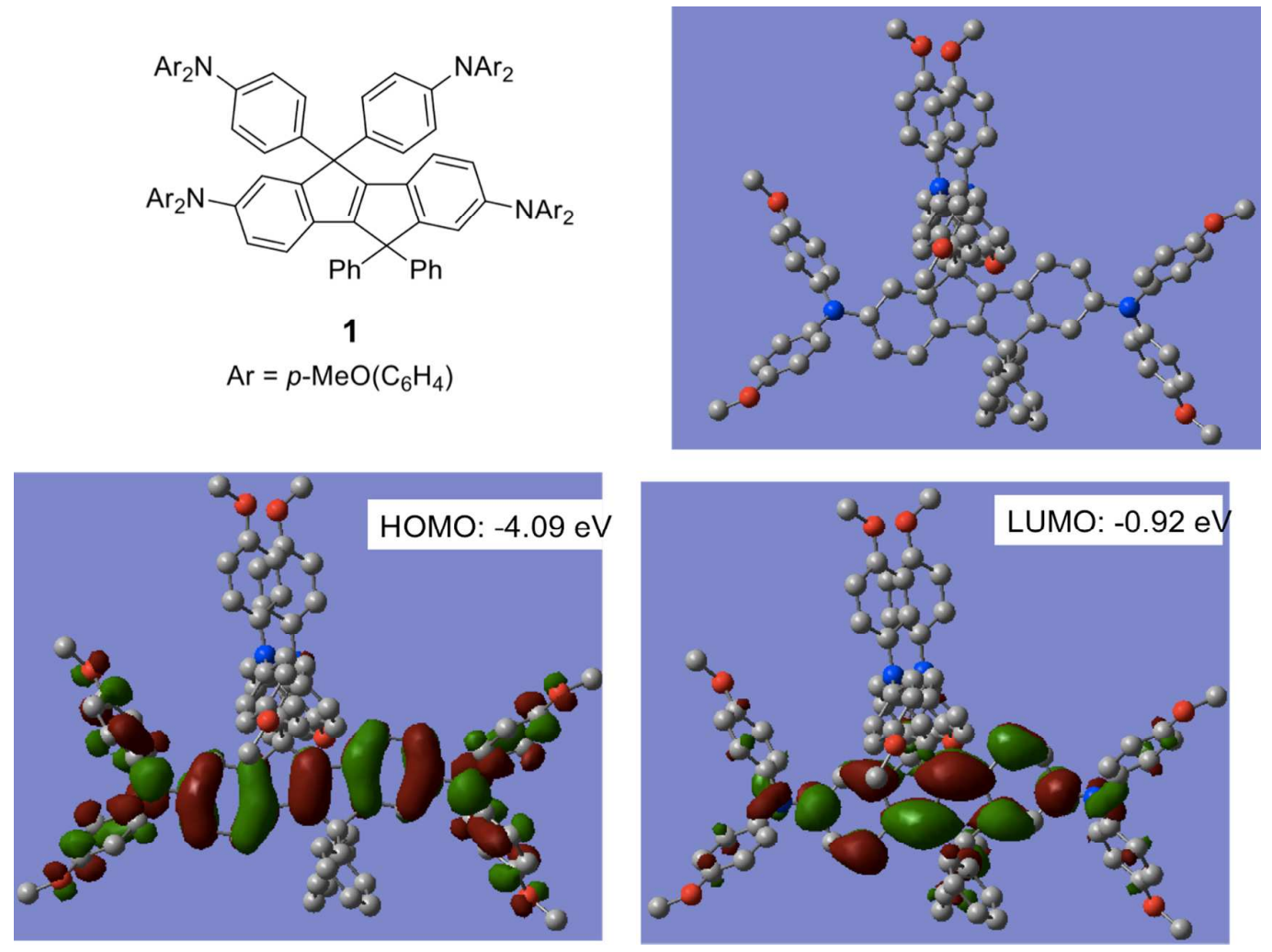

Figure S2. Chemical structure, optimized structure and FMOs of neutral 1
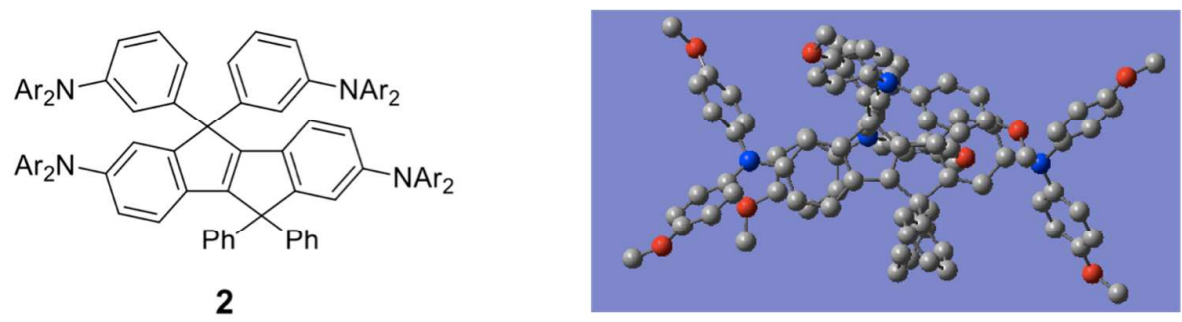

$\mathrm{Ar}=p-\mathrm{MeO}\left(\mathrm{C}_{6} \mathrm{H}_{4}\right)$
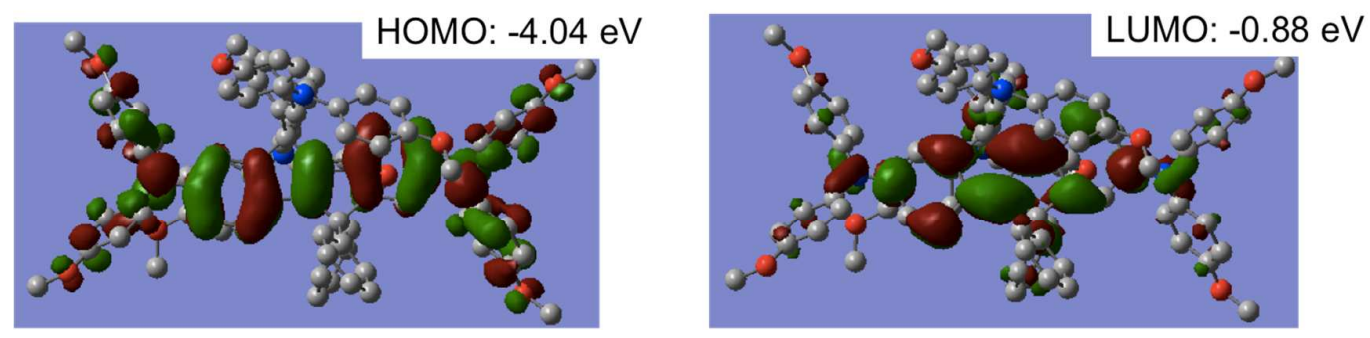

Figure S3. Chemical structure, optimized structure and FMOs of neutral 2 


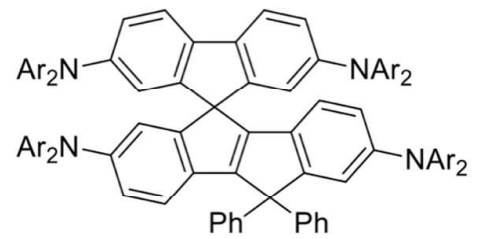

3

$\mathrm{Ar}=p-\mathrm{MeO}\left(\mathrm{C}_{6} \mathrm{H}_{4}\right)$

HOMO: $-4.04 \mathrm{eV}$

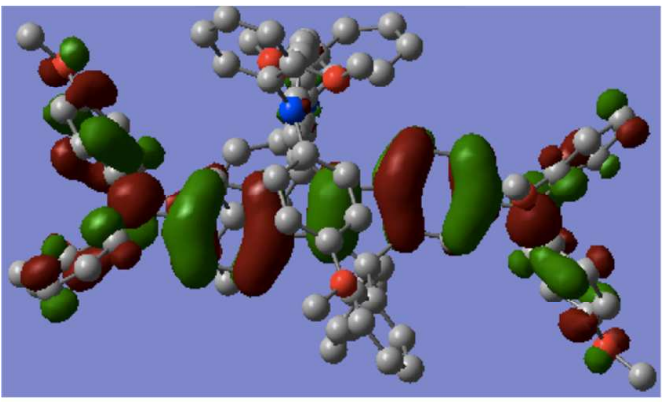

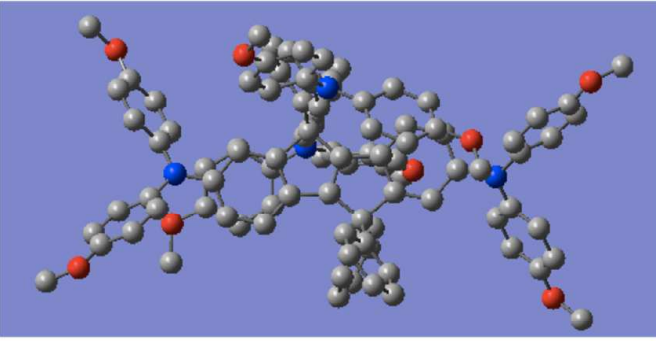

LUMO: $-0.78 \mathrm{eV}$

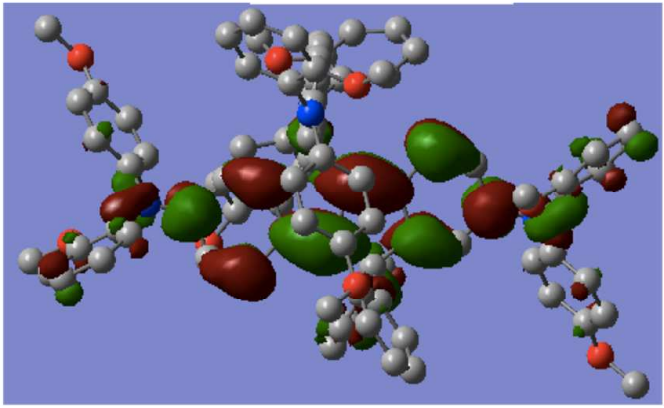

Figure S4. Chemical structure, optimized structure and FMOs of neutral 3
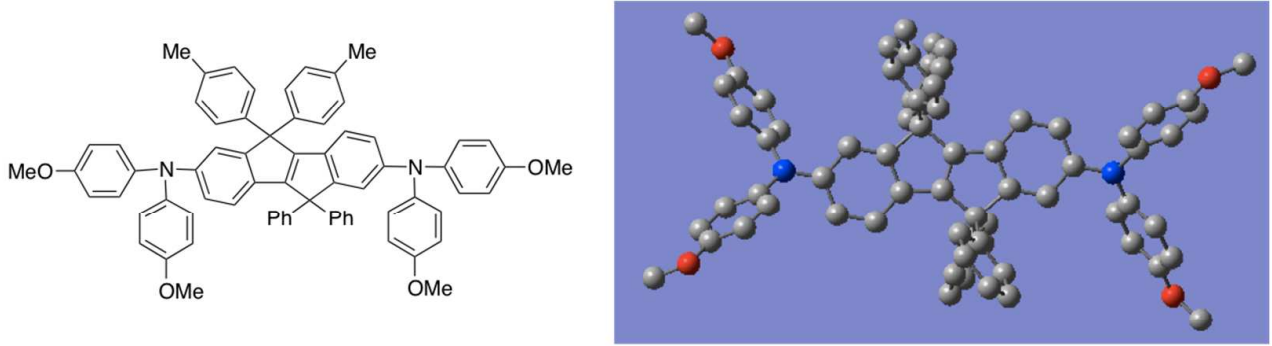

HOMO: $-4.14 \mathrm{eV}$
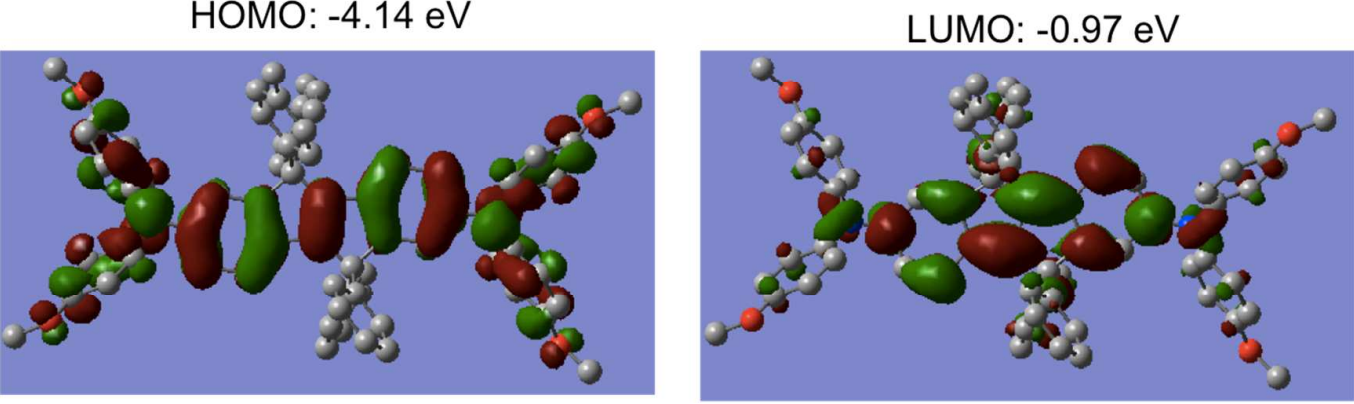

Figure S5. Chemical structure, optimized structure and FMOs of neutral 4 (octyl groups were replaced with methyl groups) 

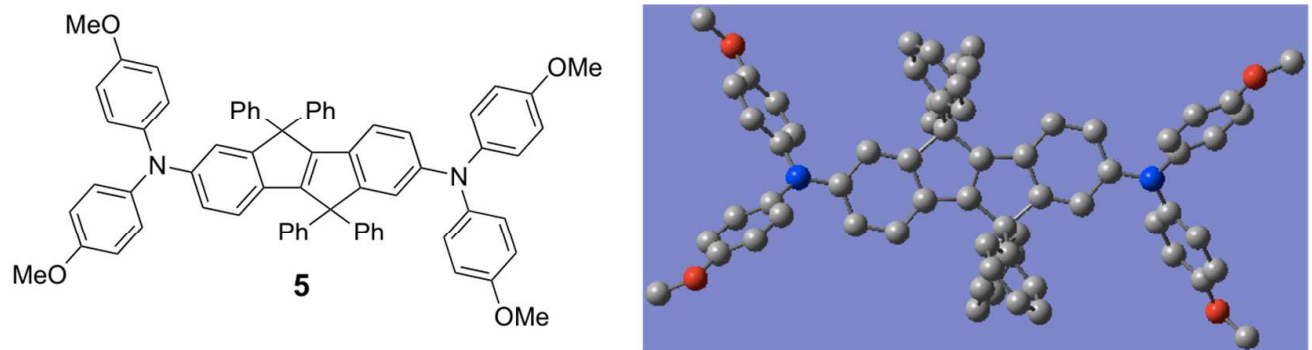

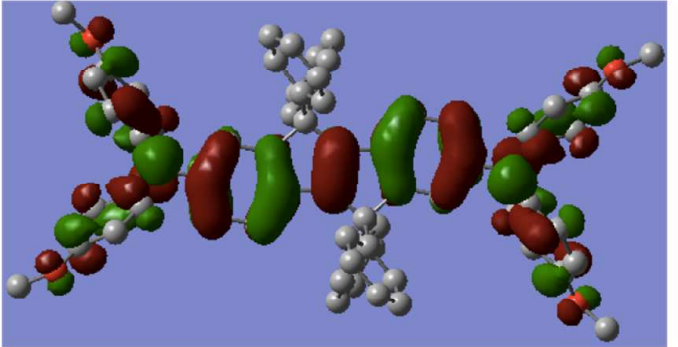

HOMO: $-4.17 \mathrm{eV}$

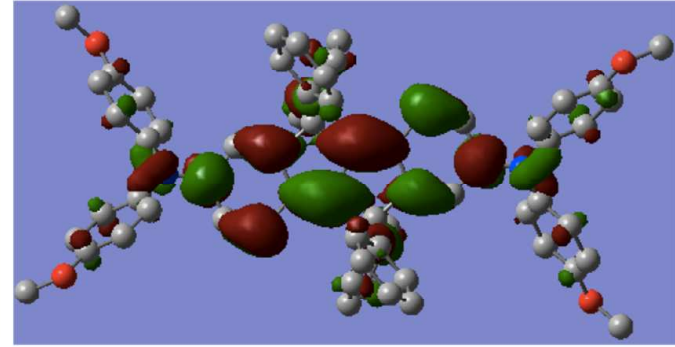

LUMO: $-1.01 \mathrm{eV}$

Figure S6. Chemical structure, optimized structure and FMOs of neutral 5 (hexyl groups were replaced with methyl groups)
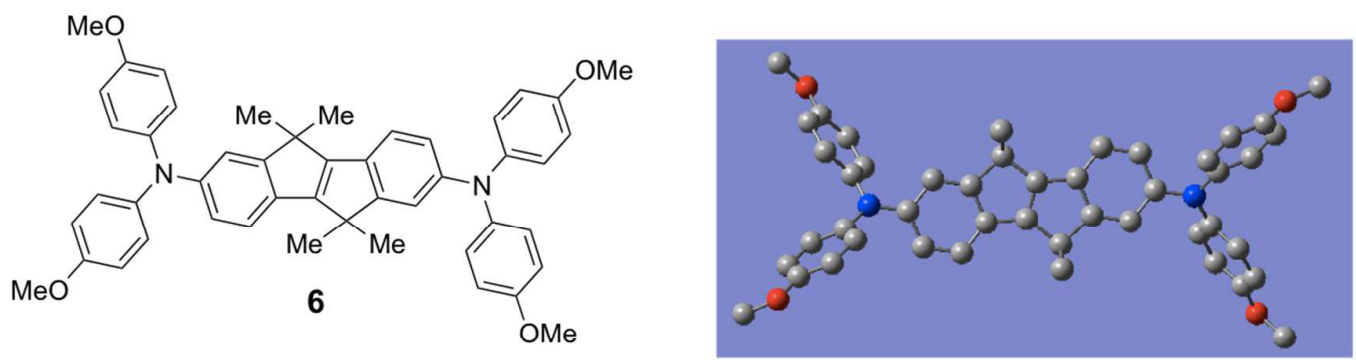

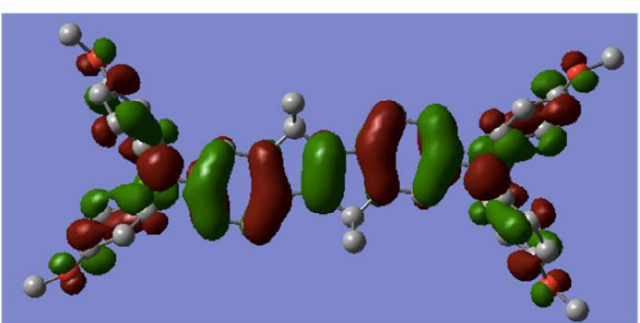

HOMO: $-4.13 \mathrm{eV}$

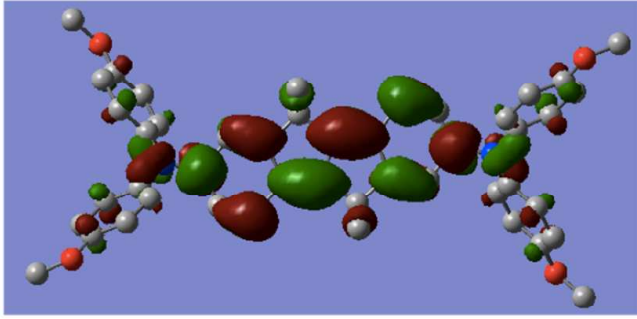

LUMO: $-0.85 \mathrm{eV}$

Figure S7. Chemical structure, optimized structure and FMOs of neutral 6 (hexyl groups were replaced with methyl groups) 


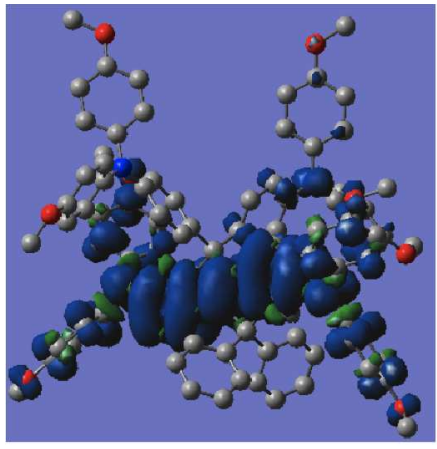

1

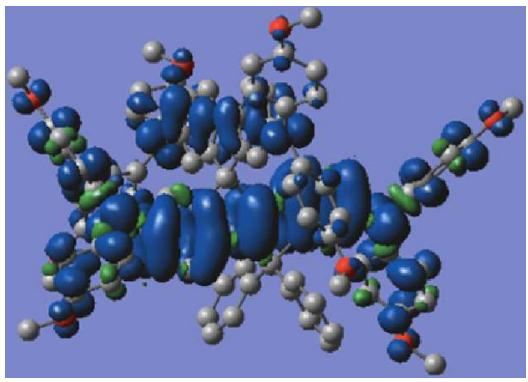

3

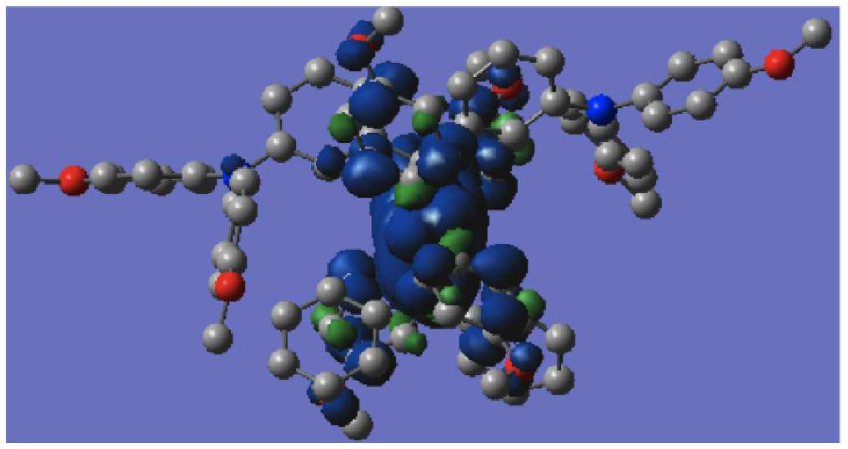

2

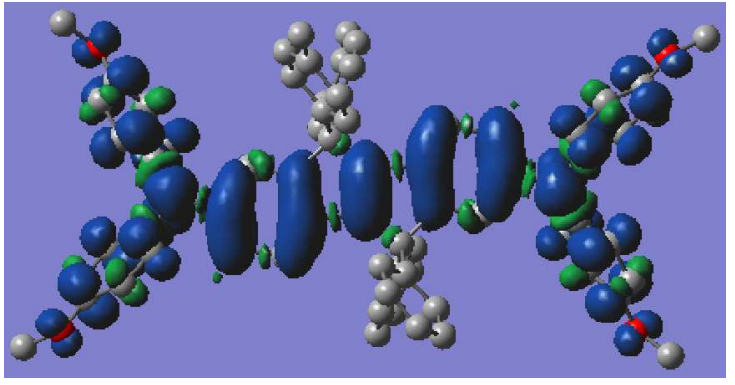

4

Figure S8. Spin density of monocation of different compounds.

Table S1 DFT calculated bond length/distance $(\AA)$ and bond angle $\left(^{\circ}\right)$ values of compound $\mathbf{1}$ in neutral and monocation states.

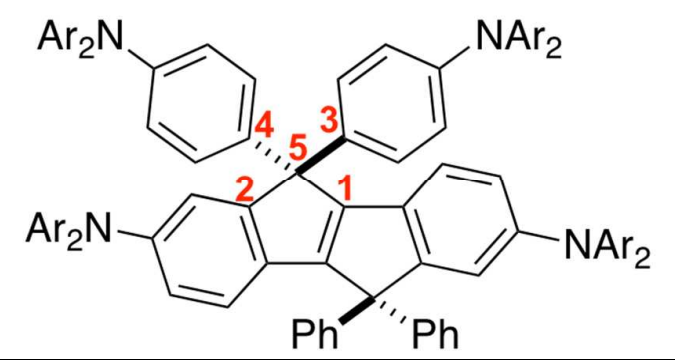

\begin{tabular}{|c|c|c|c|}
\hline & neutral & monocation & difference \\
\hline $5-1$ & 1.527 & 1.524 & -0.003 \\
\hline $5-2$ & 1.551 & 1.549 & -0.002 \\
\hline $5-3$ & 1.539 & 1.538 & -0.001 \\
\hline $5-4$ & 1.545 & 1.545 & 0 \\
\hline $1-5-3$ & 113.6 & 113.7 & 0.1 \\
\hline $1-5-4$ & 108.2 & 107.5 & -0.7 \\
\hline $2-5-3$ & 109.1 & 109.5 & 0.4 \\
\hline $2-5-4$ & 112.4 & 112.2 & -0.2 \\
\hline $1-3$ & 2.56550 & 2.56436 & -0.001 \\
\hline
\end{tabular}




\begin{tabular}{|c|c|c|c|}
\hline $1-4$ & 2.48950 & 2.47458 & -0.015 \\
\hline $2-3$ & 2.51681 & 2.52029 & 0.003 \\
\hline $2-4$ & 2.57258 & 2.56811 & -0.004 \\
\hline
\end{tabular}

Table S2 DFT calculated bond length/distance $(\AA)$ and bond angle $\left(^{\circ}\right)$ values of compound $\mathbf{2}$ in neutral and monocation states.

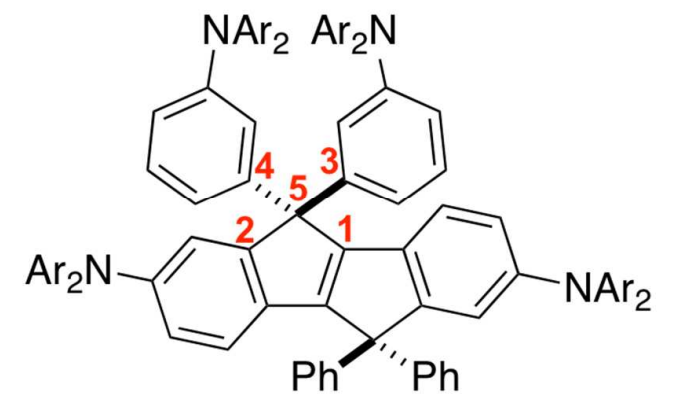

\begin{tabular}{|c|c|c|c|}
\hline & Neutral & Monocation & Difference \\
\hline $5-1$ & 1.529 & 1.526 & -0.003 \\
\hline $5-2$ & 1.551 & 1.549 & -0.002 \\
\hline $5-3$ & 1.537 & 1.538 & 0.001 \\
\hline $5-4$ & 1.551 & 1.554 & 0.003 \\
\hline $1-5-3$ & 115.5 & 115.8 & 0.3 \\
\hline $1-5-4$ & 105.7 & 105.5 & -0.2 \\
\hline $2-5-3$ & 108.8 & 109.0 & 0.2 \\
\hline $2-5-4$ & 112.6 & 112.7 & 0.1 \\
\hline $1-3$ & 2.59328 & 2.59552 & 0.002 \\
\hline $1-4$ & 2.45459 & 2.45147 & -0.003 \\
\hline $2-3$ & 2.51046 & 2.51289 & 0.002 \\
\hline $2-4$ & 2.58147 & 2.58229 & 0.001 \\
\hline
\end{tabular}

Table S3 DFT calculated bond length/distance $(\AA)$ and bond angle $\left({ }^{\circ}\right)$ values of compound $\mathbf{3}$ in neutral and monocation states.

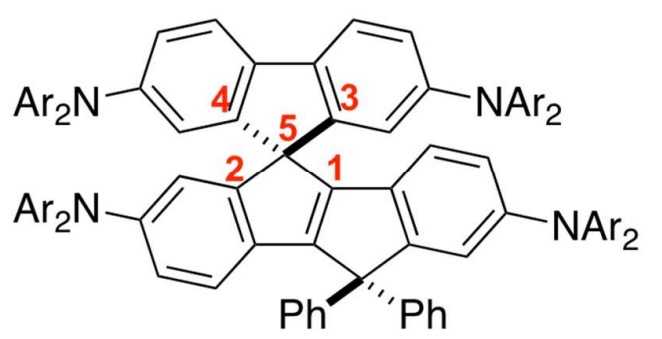

\begin{tabular}{|c|c|c|c|}
\hline & Neutral & Monocation & Difference \\
\hline $5-1$ & 1.520 & 1.518 & -0.002 \\
\hline $5-2$ & 1.537 & 1.534 & -0.003 \\
\hline
\end{tabular}




\begin{tabular}{|c|c|c|c|}
\hline $5-3$ & 1.533 & 1.535 & 0.002 \\
\hline $5-4$ & 1.535 & 1.535 & 0 \\
\hline $1-5-3$ & 114.2 & 113.5 & -0.7 \\
\hline $1-5-4$ & 112.1 & 112.0 & -0.1 \\
\hline $2-5-3$ & 114.9 & 115.1 & 0.2 \\
\hline $2-5-4$ & 114.7 & 115.0 & 0.3 \\
\hline $1-3$ & 2.56241 & 2.55323 & -0.009 \\
\hline $1-4$ & 2.53356 & 2.53062 & -0.003 \\
\hline $2-3$ & 2.58817 & 2.58910 & 0.001 \\
\hline $2-4$ & 2.58601 & 2.58889 & 0.003 \\
\hline
\end{tabular}

Table S4 DFT calculated bond length/distance $(\AA)$ and bond angle $\left({ }^{\circ}\right)$ values of compound $\mathbf{4}$ in neutral and monocation states.<smiles>CCCCNc1ccc2c(c1)C(c1ccc(C)cc1)(c1ccc(C)cc1)C1=C2c2ccc(N(CC)CC)cc2C1(c1ccccc1)c1ccccc1</smiles>

\begin{tabular}{|c|c|c|c|}
\hline & Neutral & Monocation & Difference \\
\hline $5-1$ & 1.527 & 1.525 & -0.003 \\
\hline $5-2$ & 1.551 & 1.549 & -0.002 \\
\hline $5-3$ & 1.540 & 1.541 & 0.001 \\
\hline $5-4$ & 1.546 & 1.548 & 0.002 \\
\hline $1-5-3$ & 113.7 & 113.7 & 0 \\
\hline $1-5-4$ & 108.1 & 107.9 & -0.2 \\
\hline $2-5-3$ & 109.1 & 108.9 & -0.2 \\
\hline $2-5-4$ & 112.5 & 112.3 & -0.2 \\
\hline $1-3$ & 2.56828 & 2.56730 & -0.001 \\
\hline $1-4$ & 2.48870 & 2.48437 & -0.004 \\
\hline $2-3$ & 2.51664 & 2.51442 & -0.002 \\
\hline $2-4$ & 2.57569 & 2.57534 & -0.0003 \\
\hline
\end{tabular}

Table S5 DFT calculated bond length/distance $(\AA)$ and bond angle $\left({ }^{\circ}\right)$ values of compound $\mathbf{5}$ in neutral and monocation states. (Geometry parameters are identical for C5 and C10 substituents.) 


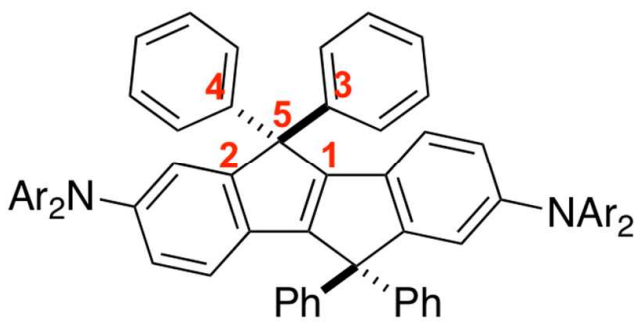

\begin{tabular}{|c|c|c|c|}
\hline & Neutral & Monocation & Difference \\
\hline $5-1$ & 1.528 & 1.525 & -0.003 \\
\hline $5-2$ & 1.551 & 1.549 & -0.002 \\
\hline $5-3$ & 1.540 & 1.541 & 0.001 \\
\hline $5-4$ & 1.547 & 1.548 & 0.001 \\
\hline $1-5-3$ & 113.9 & 113.9 & 0 \\
\hline $1-5-4$ & 107.9 & 107.8 & -0.1 \\
\hline $2-5-3$ & 109.0 & 108.8 & -0.2 \\
\hline $2-5-4$ & 112.6 & 112.6 & 0 \\
\hline $1-3$ & 2.57085 & 2.57008 & -0.001 \\
\hline $1-4$ & 2.48664 & 2.48304 & -0.004 \\
\hline $2-3$ & 2.51557 & 2.51334 & -0.002 \\
\hline $2-4$ & 2.57706 & 2.57719 & 0.0001 \\
\hline
\end{tabular}

Table S6 DFT calculated bond length/distance $(\AA)$ and bond angle $\left({ }^{\circ}\right)$ values of compound $\mathbf{6}$ in neutral and monocation states. (Geometry parameters are identical for C5 and C10 substituents.)<smiles>CCCCNc1ccc2c(c1)C(C)(C)C1=C2c2ccc(N(C)CC)cc2C1(C)C</smiles>

\begin{tabular}{|c|c|c|c|}
\hline & Neutral & Monocation & Difference \\
\hline $5-1$ & 1.518 & 1.516 & -0.002 \\
\hline $5-2$ & 1.537 & 1.536 & -0.001 \\
\hline $5-3$ & 1.545 & 1.546 & 0.001 \\
\hline $5-4$ & 1.545 & 1.546 & 0.001 \\
\hline $1-5-3$ & 111.8 & 111.8 & 0 \\
\hline $1-5-4$ & 111.8 & 111.8 & 0 \\
\hline $2-5-3$ & 111.4 & 111.4 & 0 \\
\hline $2-5-4$ & 111.2 & 111.3 & 0.001 \\
\hline $1-3$ & 2.53647 & 2.53534 & -0.001 \\
\hline $1-4$ & 2.53656 & 2.53642 & -0.0001 \\
\hline
\end{tabular}




\begin{tabular}{|c|c|c|c|}
\hline $2-3$ & 2.54613 & 2.54655 & 0.0004 \\
\hline $2-4$ & 2.54262 & 2.54397 & 0.001 \\
\hline
\end{tabular}

\section{Energy levels, film XRD, UV-vis spectra, morphology analysis and J-V} characteristics of HTLs

Table S7. The energy levels of different compounds.

\begin{tabular}{ccccccccc}
\hline Compounds & 1 & 2 & 3 & 4 & 5 & 6 & 7 & spiro-OMeTAD \\
\hline HOMO/eV & -4.9 & -4.9 & -4.9 & -4.9 & -4.9 & -4.8 & -5.3 & -5.2 \\
\hline Band gap/eV & 2.7 & 2.7 & 2.7 & 2.7 & 2.7 & 2.8 & 3.0 & 2.9 \\
\hline
\end{tabular}

The hole mobilities were measured by the space charge limited current (SCLC) method with a device configuration of ITO/PEDOT:PSS/HTL(or HTL-doped)/Au. All HTLs were prepared with the same conditions as for photovoltaic devices. The SCLC is described by $J=(9 / 8) \varepsilon_{0} \varepsilon_{\mathrm{r}} \mu\left(\mathrm{V}^{2} / \mathrm{L}^{3}\right)$, Where $\varepsilon_{0}$ is permittivity of free space, $\varepsilon_{0}$ is dielectric constant vacuum. $\mu$ hole is hole mobility, $\mathrm{V}$ is voltage drop across the device and L is thickness of HTLs. The dielectric constant $\varepsilon_{\mathrm{r}}$ is assumed to be 3 , which is a typical value for spiro-like organic HTLs. ${ }^{\text {S }}$

Table S8, Details of mobility and conductivity of doped and undoped compounds 1-7 and spiro-MeOTAD.

\begin{tabular}{ccccc}
\hline Compound & $\begin{array}{c}\text { Mobility } \\
\left(\mathrm{cm}^{2} \mathrm{~V}^{-1} \mathrm{~s}^{-1}\right)\end{array}$ & $\begin{array}{c}\text { Conductivity } \\
(\mathrm{S} / \mathrm{cm})\end{array}$ & $\begin{array}{c}\text { Mobility (doped) } \\
\left(\mathrm{cm}^{2} \mathrm{~V}^{-1} \mathrm{~s}^{-1}\right)\end{array}$ & $\begin{array}{c}\text { Conductivity (doped) } \\
(\mathrm{S} / \mathrm{cm})\end{array}$ \\
\hline $\mathbf{1}$ & $1.1 \times 10^{-5}$ & $2.9 \times 10^{-7}$ & $3.9 \times 10^{-5}$ & $3.7 \times 10^{-6}$ \\
$\mathbf{2}$ & $0.7 \times 10^{-5}$ & $2.2 \times 10^{-7}$ & $1.5 \times 10^{-5}$ & $1.3 \times 10^{-6}$ \\
$\mathbf{3}$ & $1.4 \times 10^{-5}$ & $2.7 \times 10^{-7}$ & $2.1 \times 10^{-5}$ & $1.9 \times 10^{-6}$ \\
$\mathbf{4}$ & $0.3 \times 10^{-5}$ & $1.0 \times 10^{-7}$ & $4.7 \times 10^{-5}$ & $0.7 \times 10^{-6}$ \\
$\mathbf{5}$ & $1.2 \times 10^{-5}$ & $1.6 \times 10^{-7}$ & $4.3 \times 10^{-5}$ & $1.4 \times 10^{-6}$ \\
$\mathbf{6}$ & $0.5 \times 10^{-5}$ & $0.2 \times 10^{-7}$ & $0.8 \times 10^{-5}$ & $0.8 \times 10^{-6}$ \\
$\mathbf{7}$ & $0.3 \times 10^{-5}$ & $0.7 \times 10^{-7}$ & $2.6 \times 10^{-5}$ & $1.3 \times 10^{-6}$ \\
spiro-MeOTAD & $2.6 \times 10^{-5}$ & $5.6 \times 10^{-7}$ & $2.6 \times 10^{-5}$ & $2.0 \times 10^{-6}$ \\
\hline
\end{tabular}



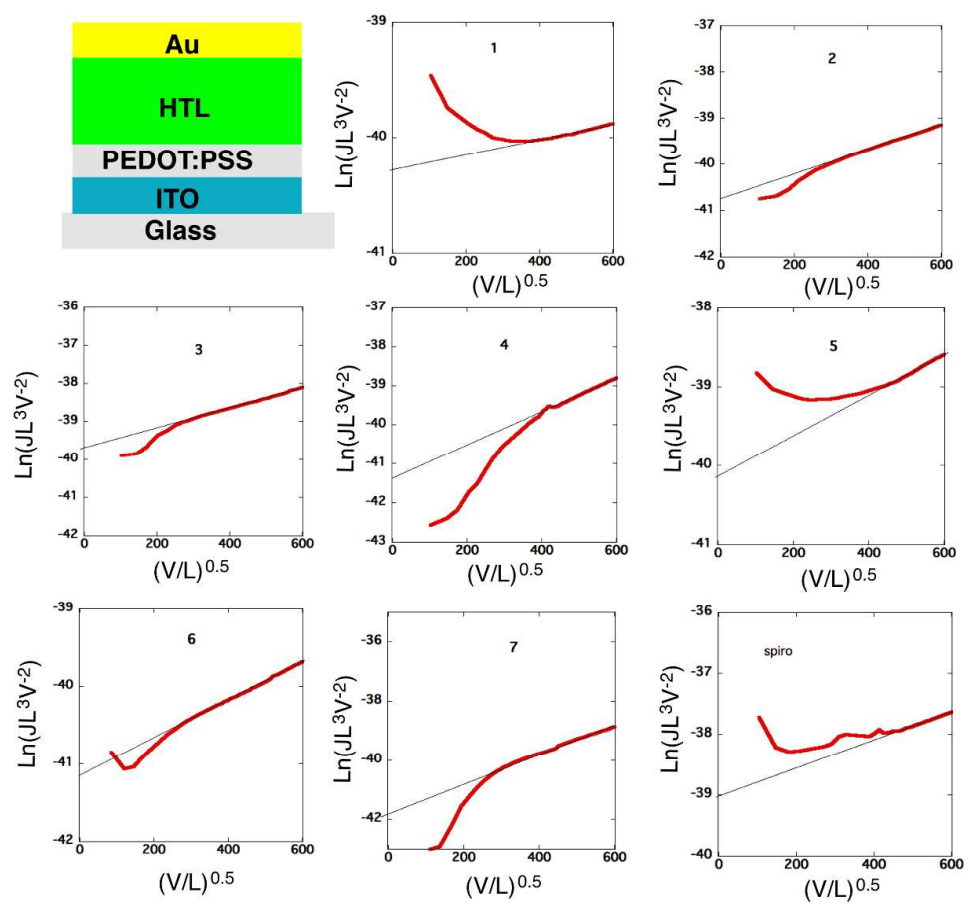

Figure S9. J-V curves of hole-only transporting devices based on different compounds.
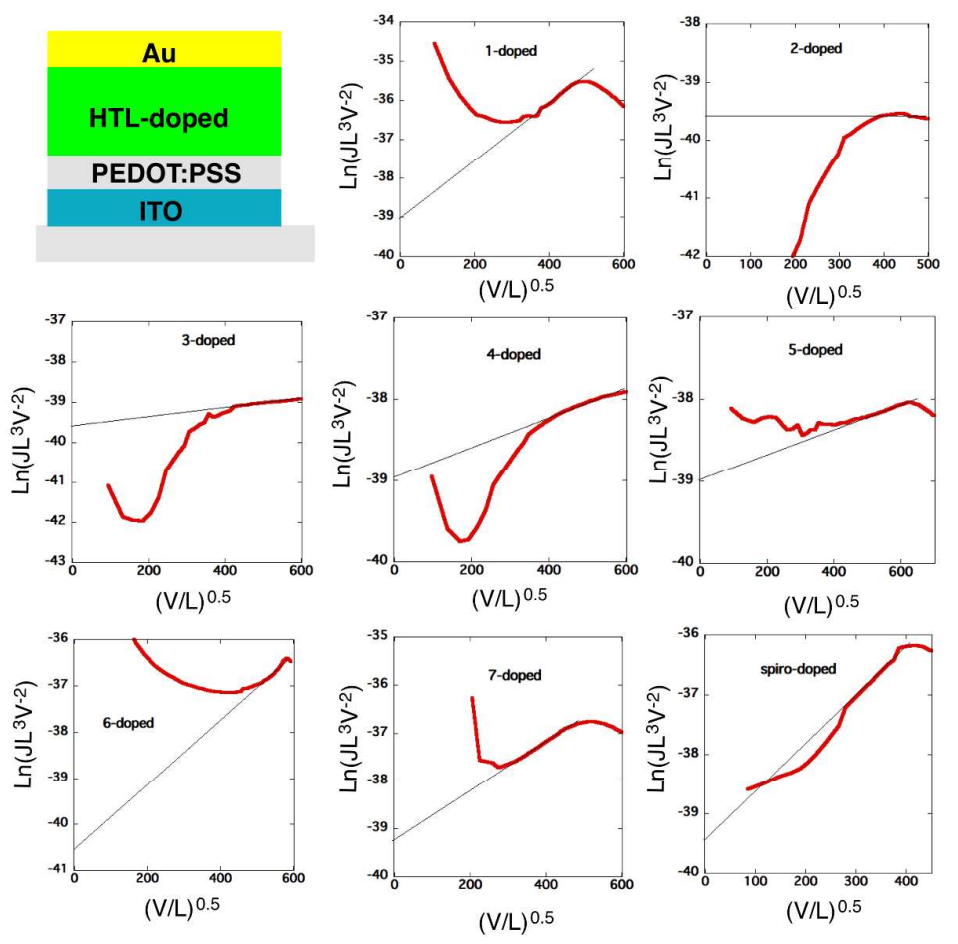

Figure S10. J-V curves of hole-only transporting devices based on doped different compounds. 

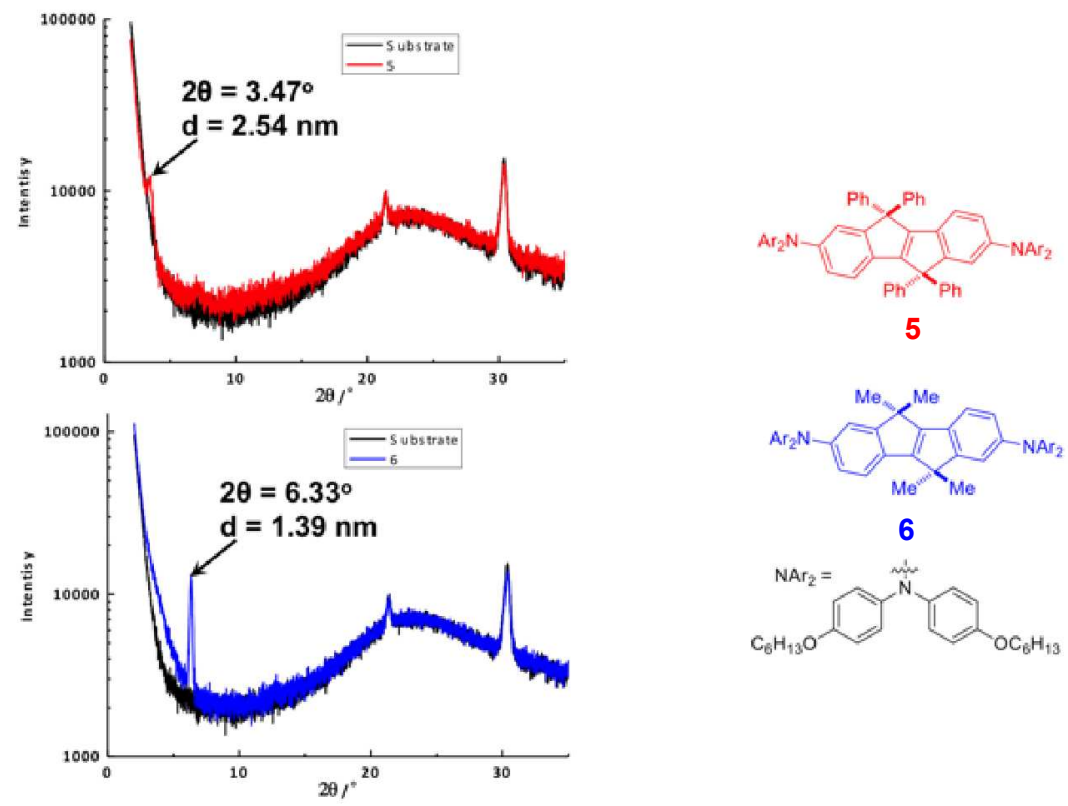

Figure S11. XRD patterns of films of $\mathbf{5}$ and $\mathbf{6}$ on ITO substrates. (Diffraction peaks are labeled.)
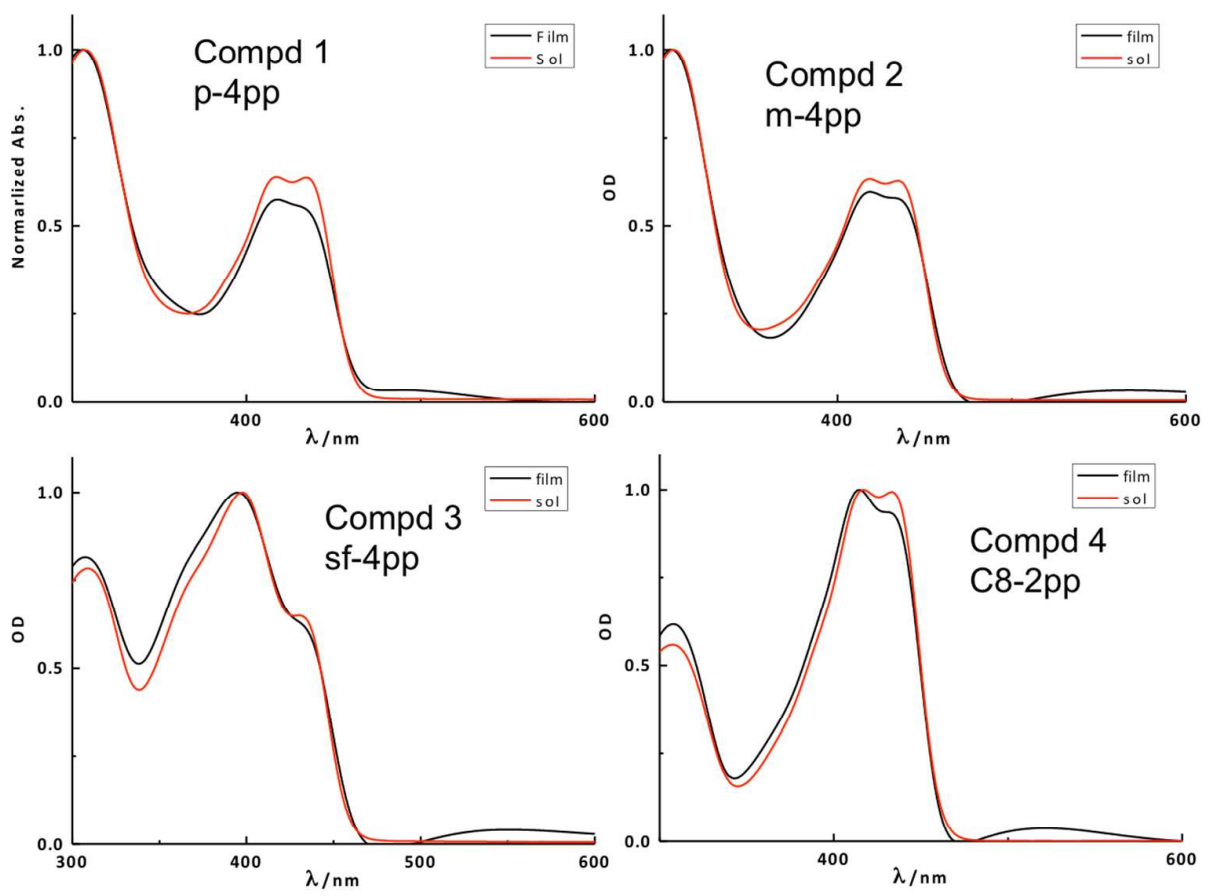

Figure S12. Comparison of absorption spectra of compounds 1-4 in solution and film. 


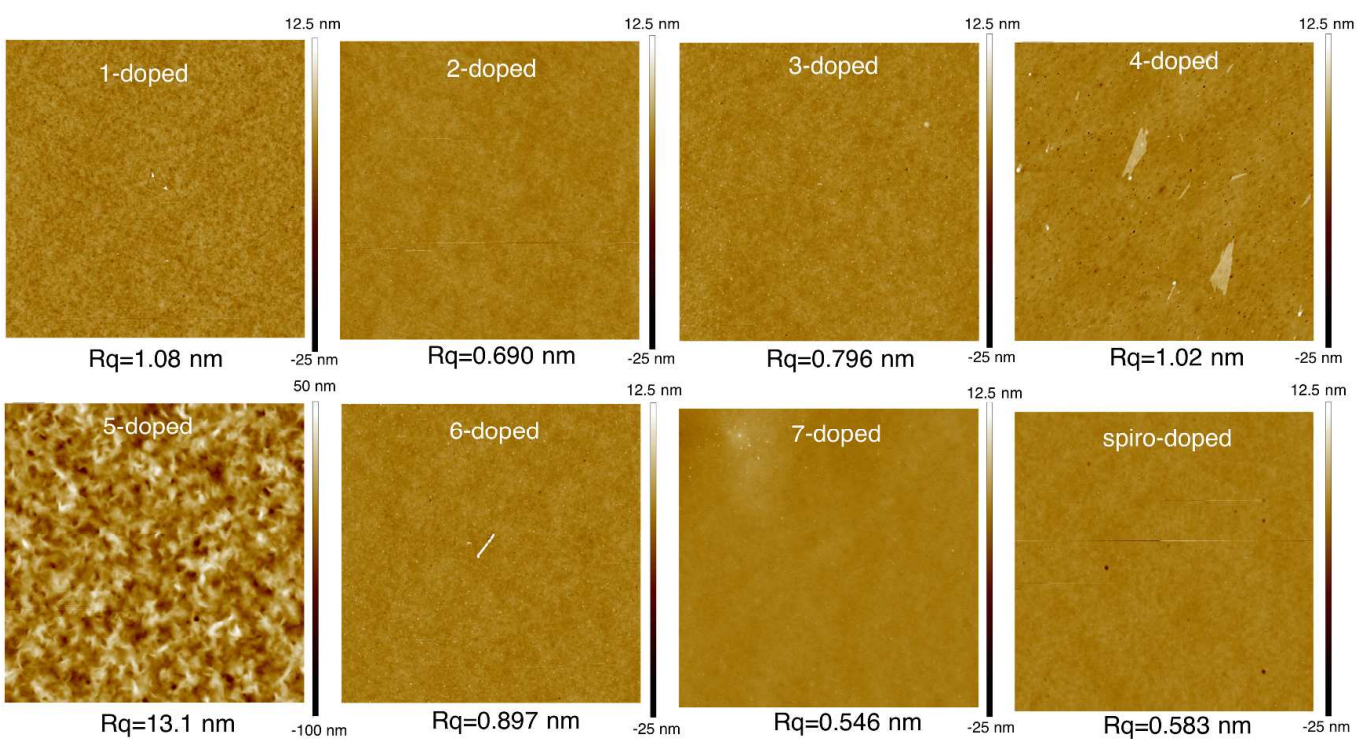

Figure S13, AFM images of different HTL films on PEDOT:PSS/ITO/glass surface. The label (e.g., n-doped) refers to the data for doped compound n (1-7). spiro refers to spiro-MeOTAD. 


\section{References:}

(S1) (a) A. D. Becke, Phys. Rev. A, 1988, 38, 3098-3100. (b) C. Lee, W. Yang, G. G. Parr, Phys. Rev. B. 1988, 37, 785-789.

(S2) M. J. Frisch, G. W. Trucks, H. B. Schlegel, G. E. Scuseria, M. A. Robb, J. R.

Cheeseman, G. Scalmani, V. Barone, B. Mennucci, G. A. Petersson, H. Nakatsuji, M.

Caricato, X. Li, H. P. Hratchian, A. F. Izmaylov, J. Bloino, G. Zheng, J. L.

Sonnenberg, M. Hada, M. Ehara, K. Toyota, R. Fukuda, J. Hasegawa, M. Ishida, T.

Nakajima, Y. Honda, O. Kitao, H. Nakai, T. Vreven, J. A. Montgomery, Jr., J. E.

Peralta, F. Ogliaro, M. Bearpark, J. J. Heyd, E. Brothers, K. N. Kudin, V. N.

Staroverov, T. Keith, R. Kobayashi, J. Normand, K. Raghavachari, A. Rendell, J. C.

Burant, S. S. Iyengar, J. Tomasi, M. Cossi, N. Rega, J. M. Millam, M. Klene, J. E.

Knox, J. B. Cross, V. Bakken, C. Adamo, J. Jaramillo, R. Gomperts, R. E. Stratmann, O. Yazyev, A. J. Austin, R. Cammi, C. Pomelli, J. W. Ochterski, R. L. Martin, K.

Morokuma, V. G. Zakrzewski, G. A. Voth, P. Salvador, J. J. Dannenberg, S. Dapprich, A. D. Daniels, O. Farkas, J. B. Foresman, J. V. Ortiz, J. Cioslowski, and D. J. Fox, Gaussian, Inc., Wallingford CT, 2010.) and the orbital pictures were prepared using Gaussview.( R. II Dennington, T. Keith, J. Millam, K. Eppinnett, W. L. Hovell, R. Gilliland, GaussView, Version 3.09; Semichem, Inc.: Shawnee Mission, KS, 2003. (S3) a) Yin, J.; Chen, R. -F.; Zhang, S. -L.; Ling, Q. -D.; Huang, W. J. Phys. Chem. A 2010, 114, 3655-3667. b) Buda, M. Electrochim. Acta 2013, 113, 536-549.

(S4) Leijtens, T.; Ding, I-K.; Giovenzana, T.; Bloking, J.; McGehee, M.; Sellinger, A.; ACSNano, 2012, 6, 1455-1462. 


\section{NMR charts}

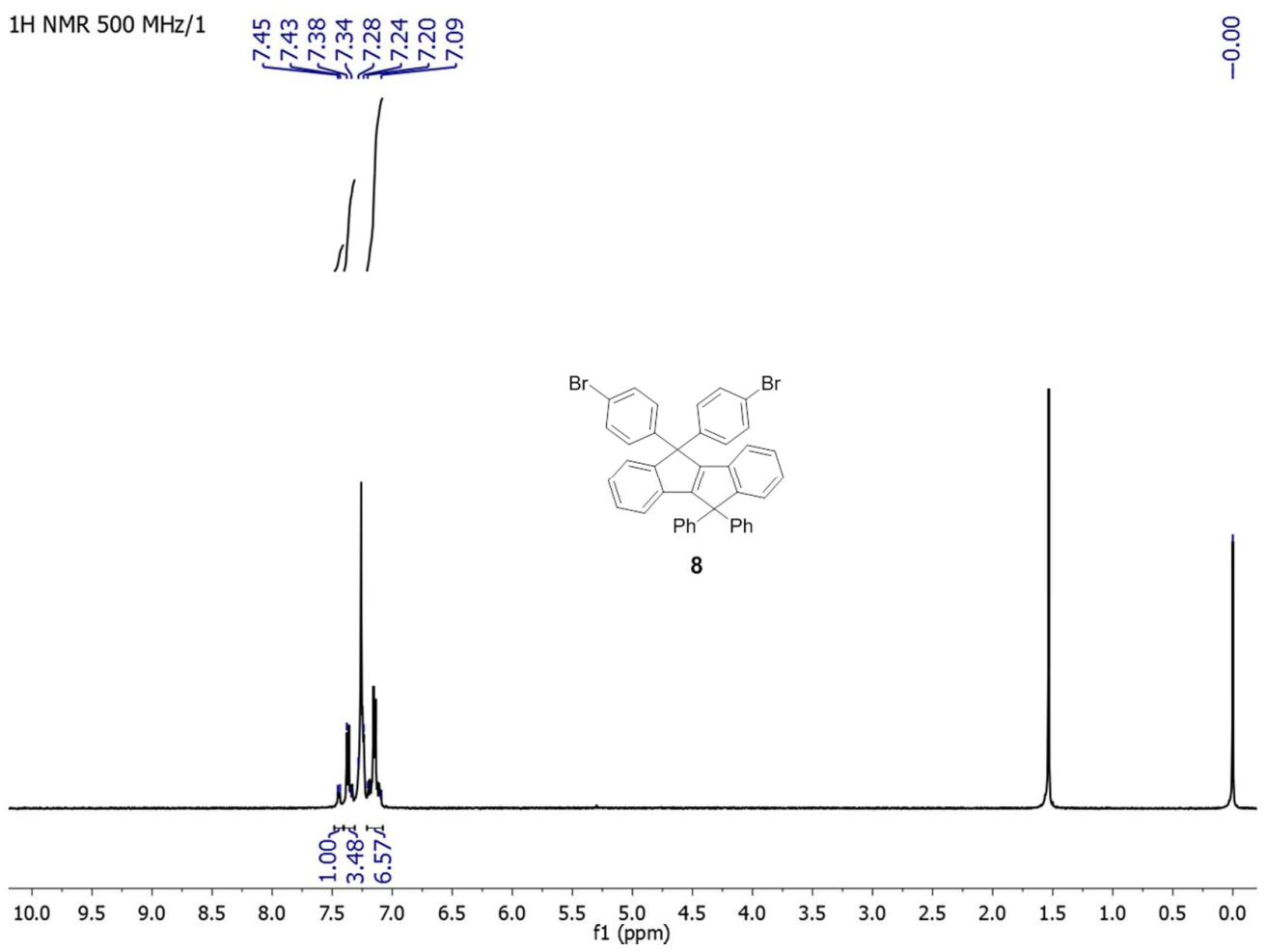

${ }^{1} \mathrm{H}$ NMR spectrum of $\mathbf{1 0}$

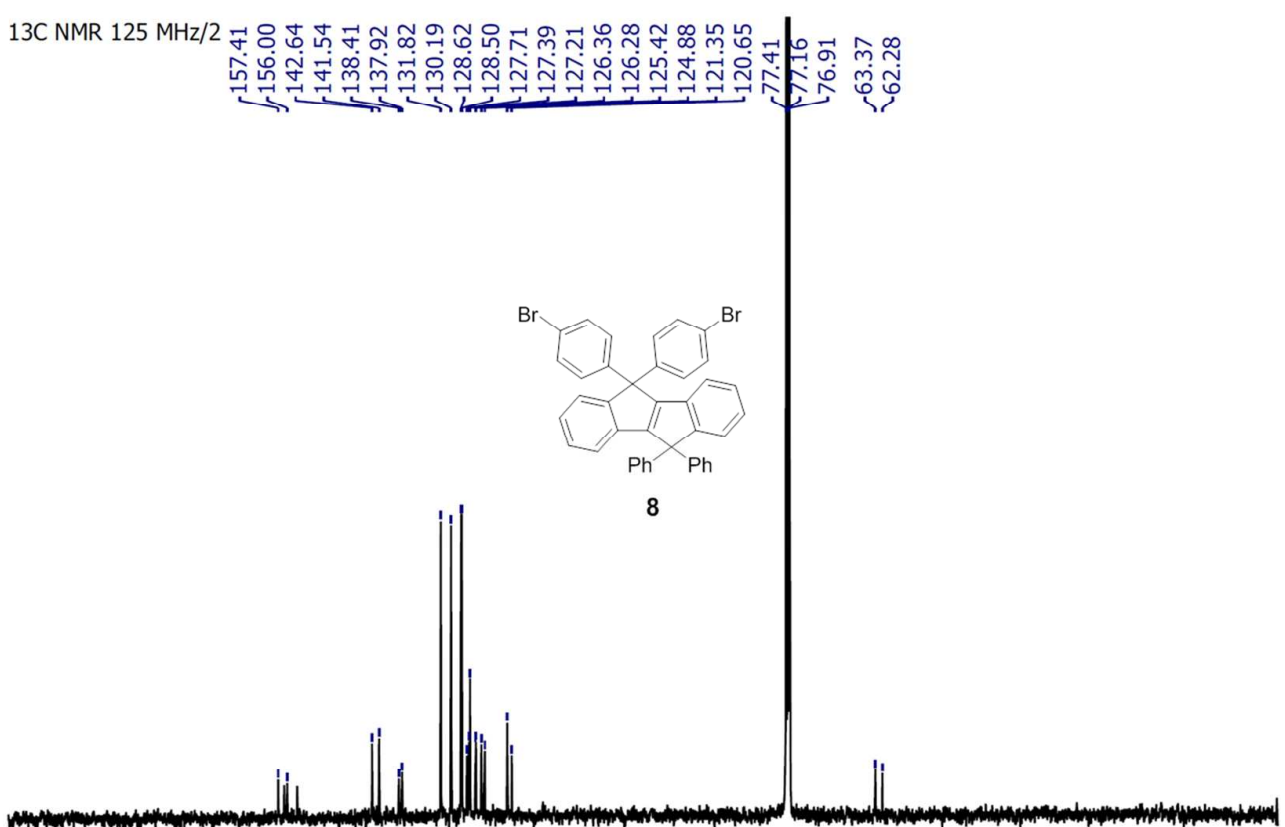

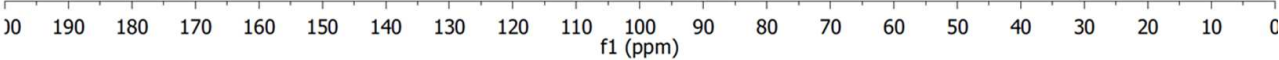

${ }^{13} \mathrm{C}$ NMR spectrum of $\mathbf{1 0}$ 


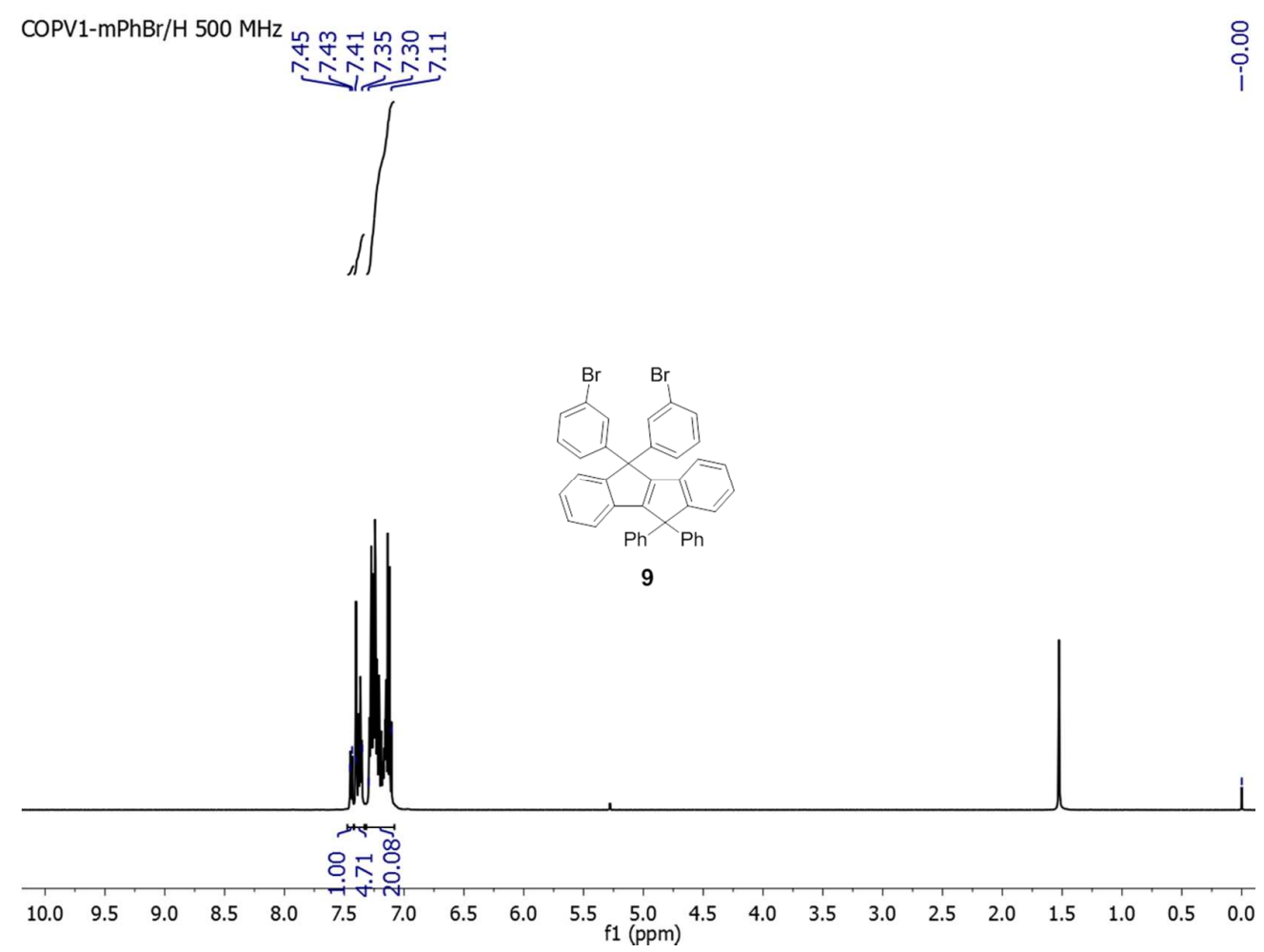

${ }^{1} \mathrm{H}$ NMR spectrum of $\mathbf{1 1}$

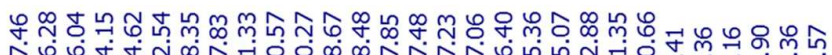

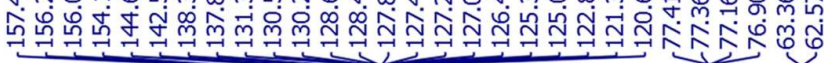
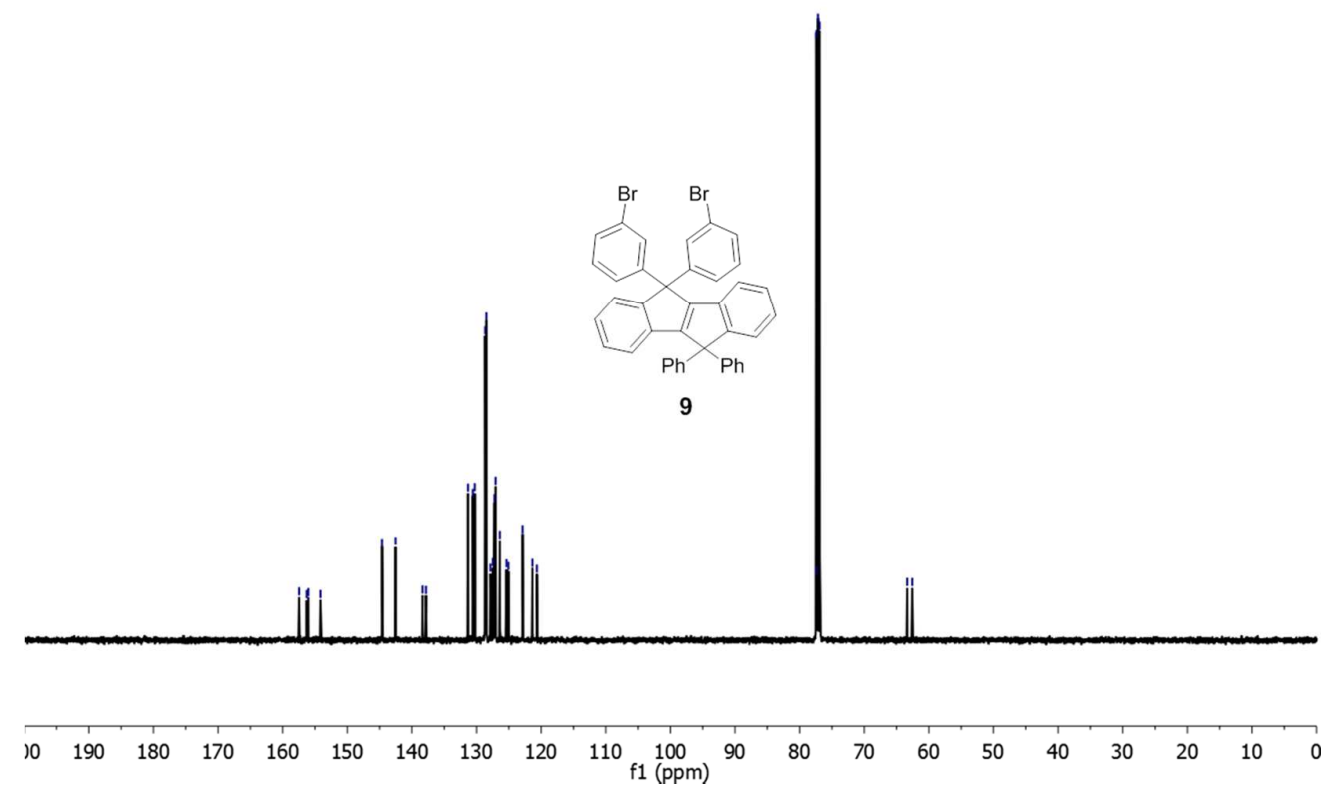

${ }^{13} \mathrm{C}$ NMR spectrum of $\mathbf{1 1}$ 


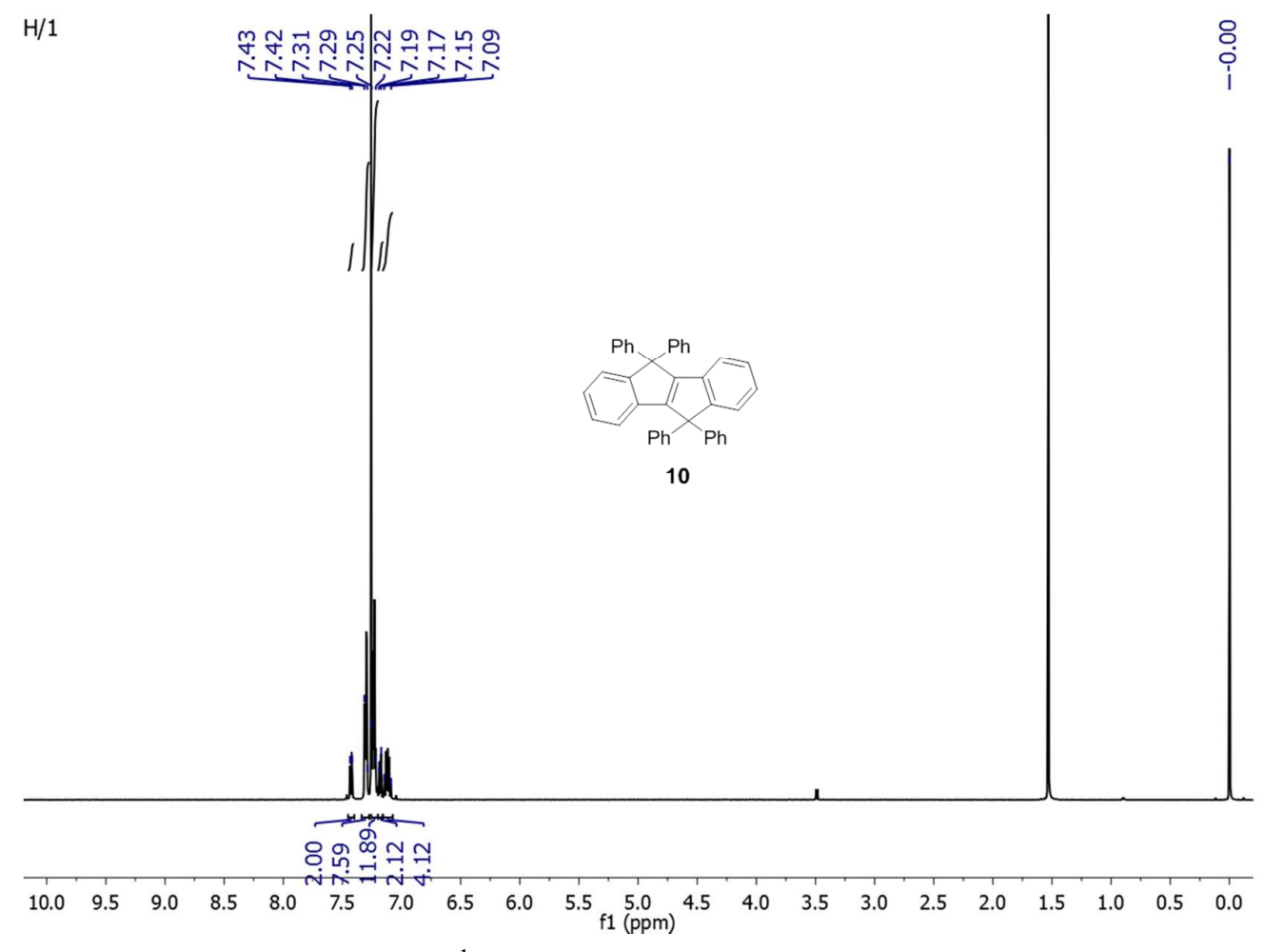

${ }^{1} \mathrm{H}$ NMR spectrum of $\mathbf{1 2}$

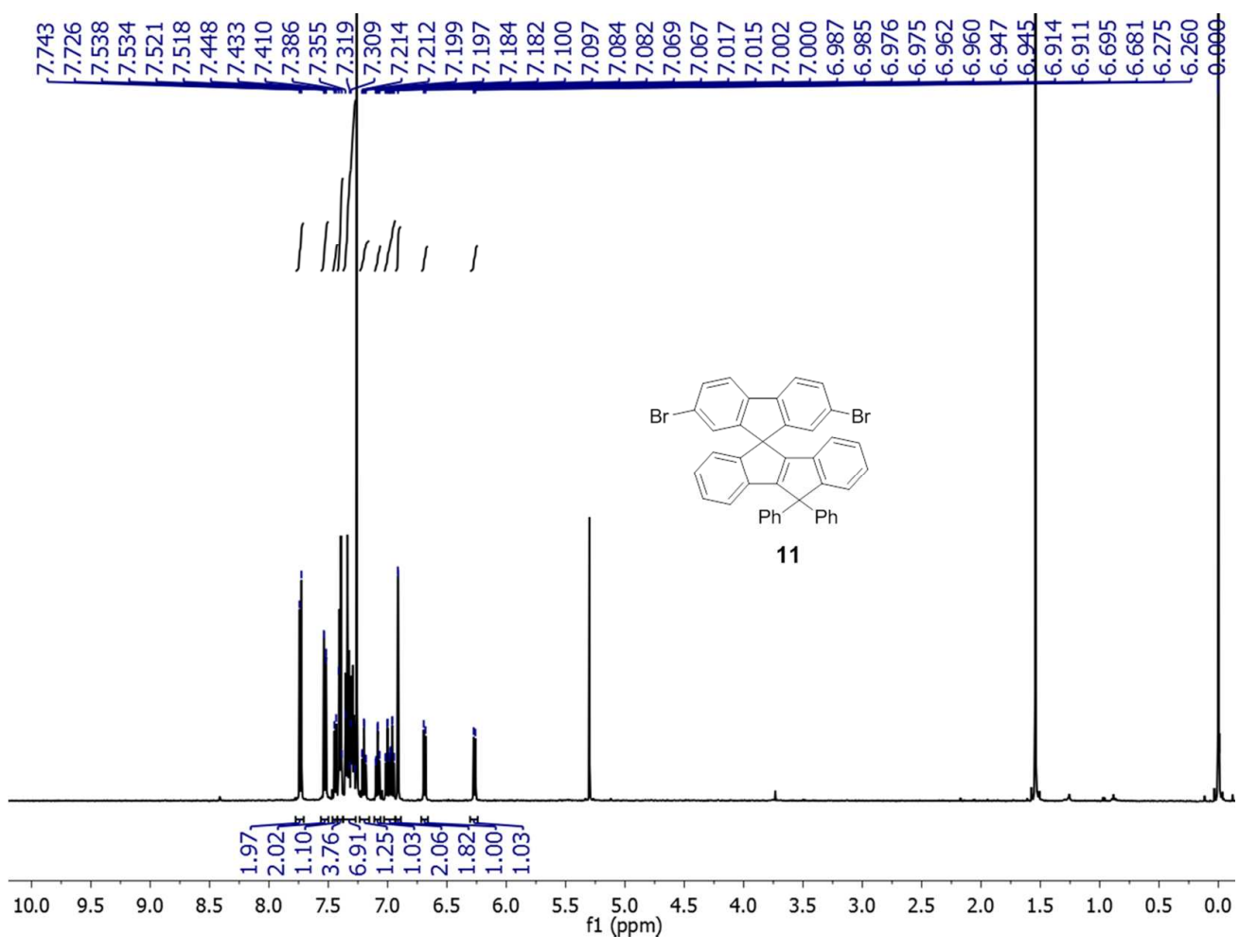

${ }^{1} \mathrm{H}$ NMR spectrum of $\mathbf{1 3}$ 


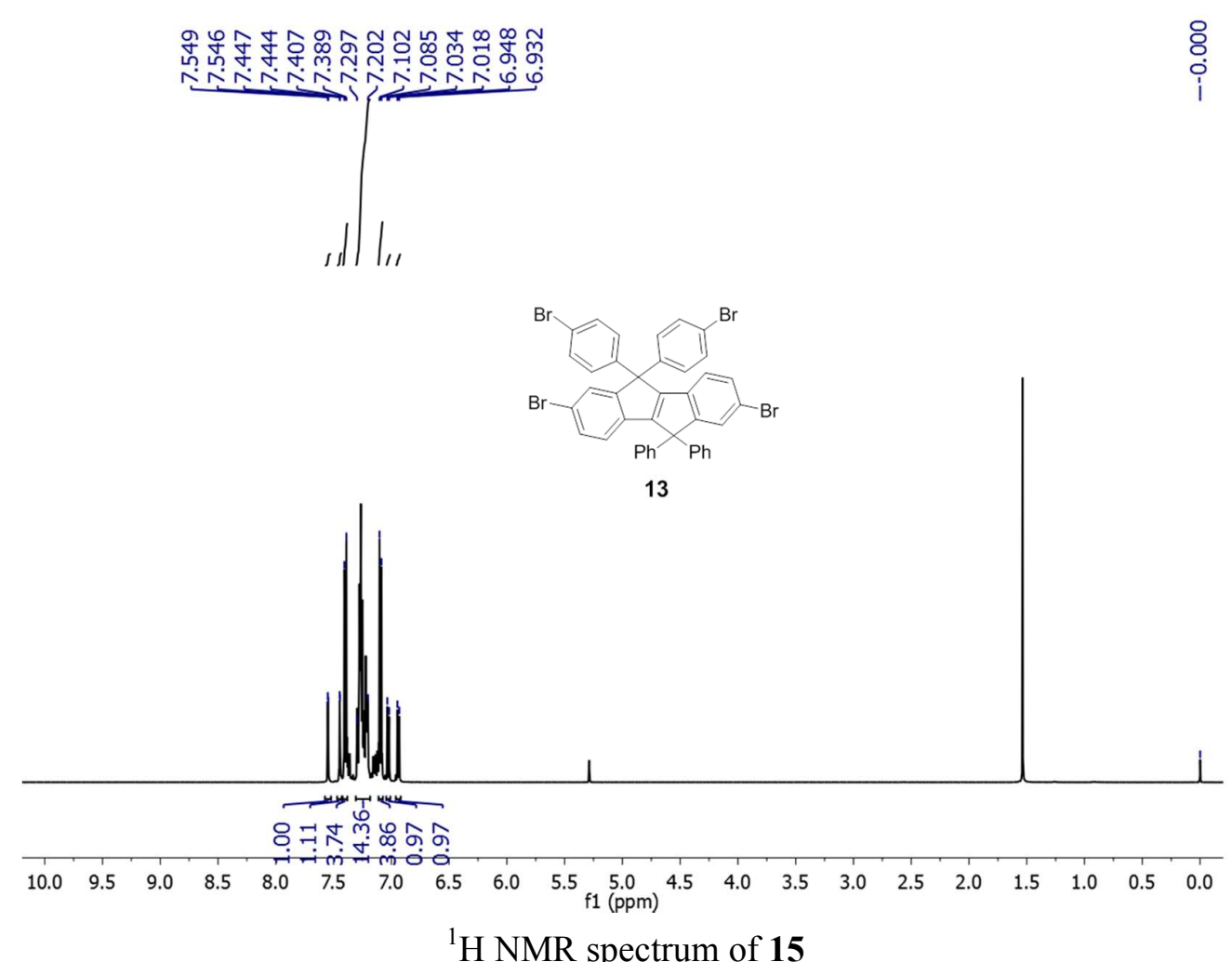

${ }^{1} \mathrm{H}$ NMR spectrum of $\mathbf{1 5}$

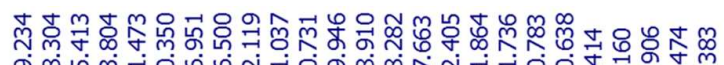

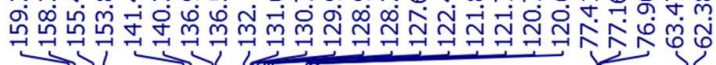

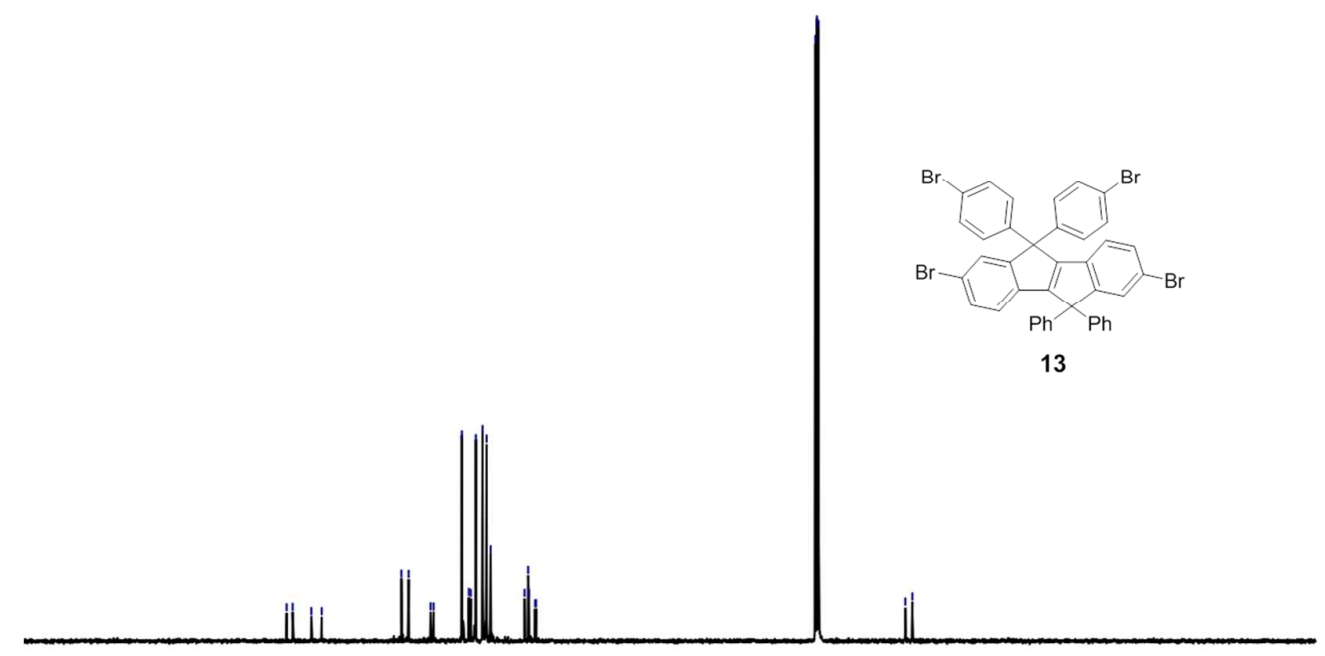

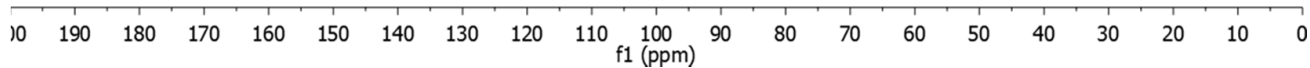

${ }^{13} \mathrm{C}$ NMR spectrum of $\mathbf{1 5}$ 

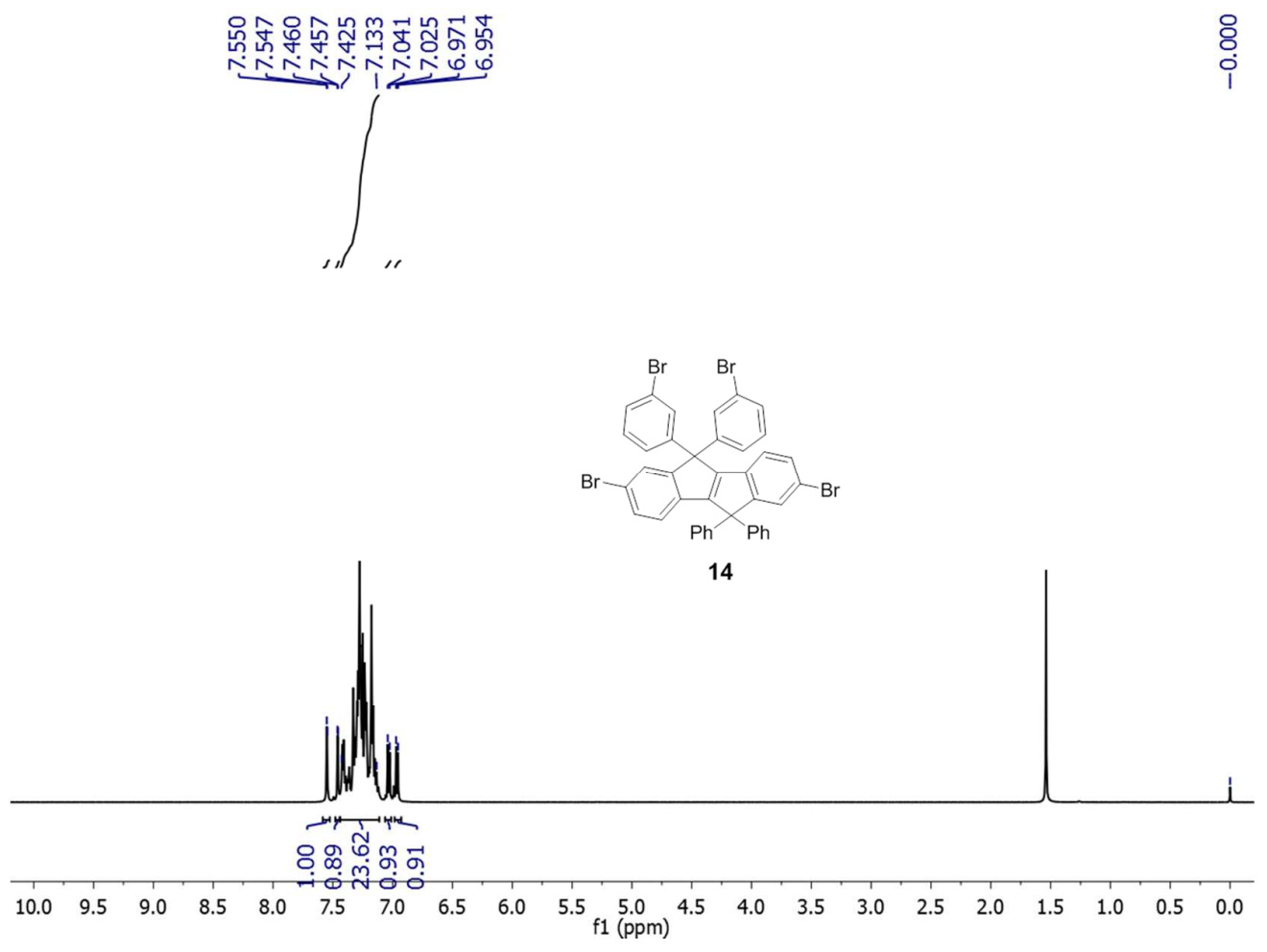

${ }^{1} \mathrm{H}$ NMR spectrum of $\mathbf{1 6}$

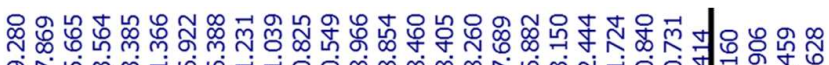

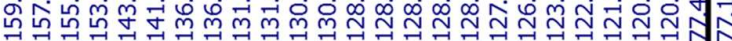

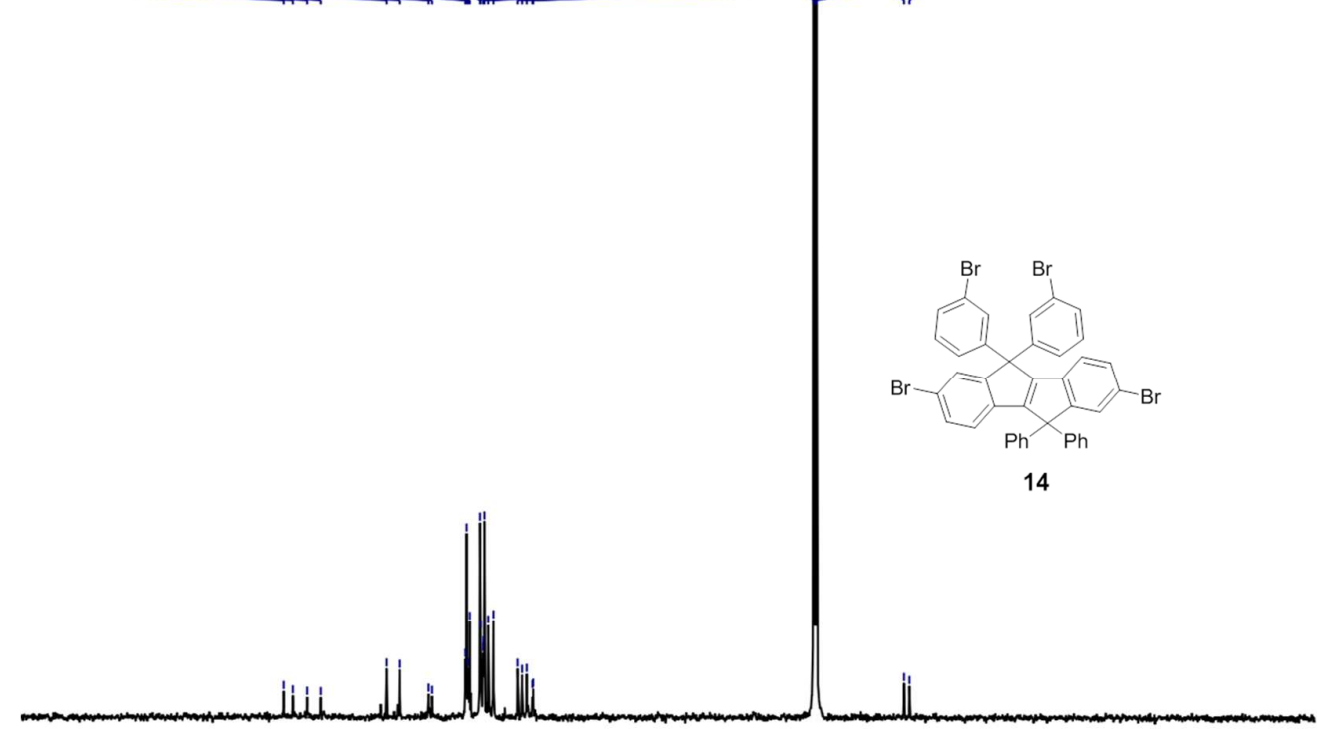

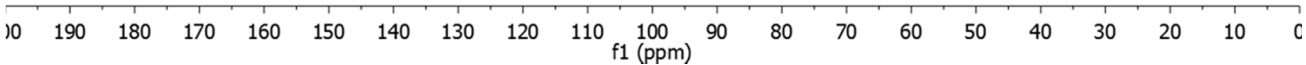

${ }^{13} \mathrm{C}$ NMR spectrum of $\mathbf{1 6}$ 


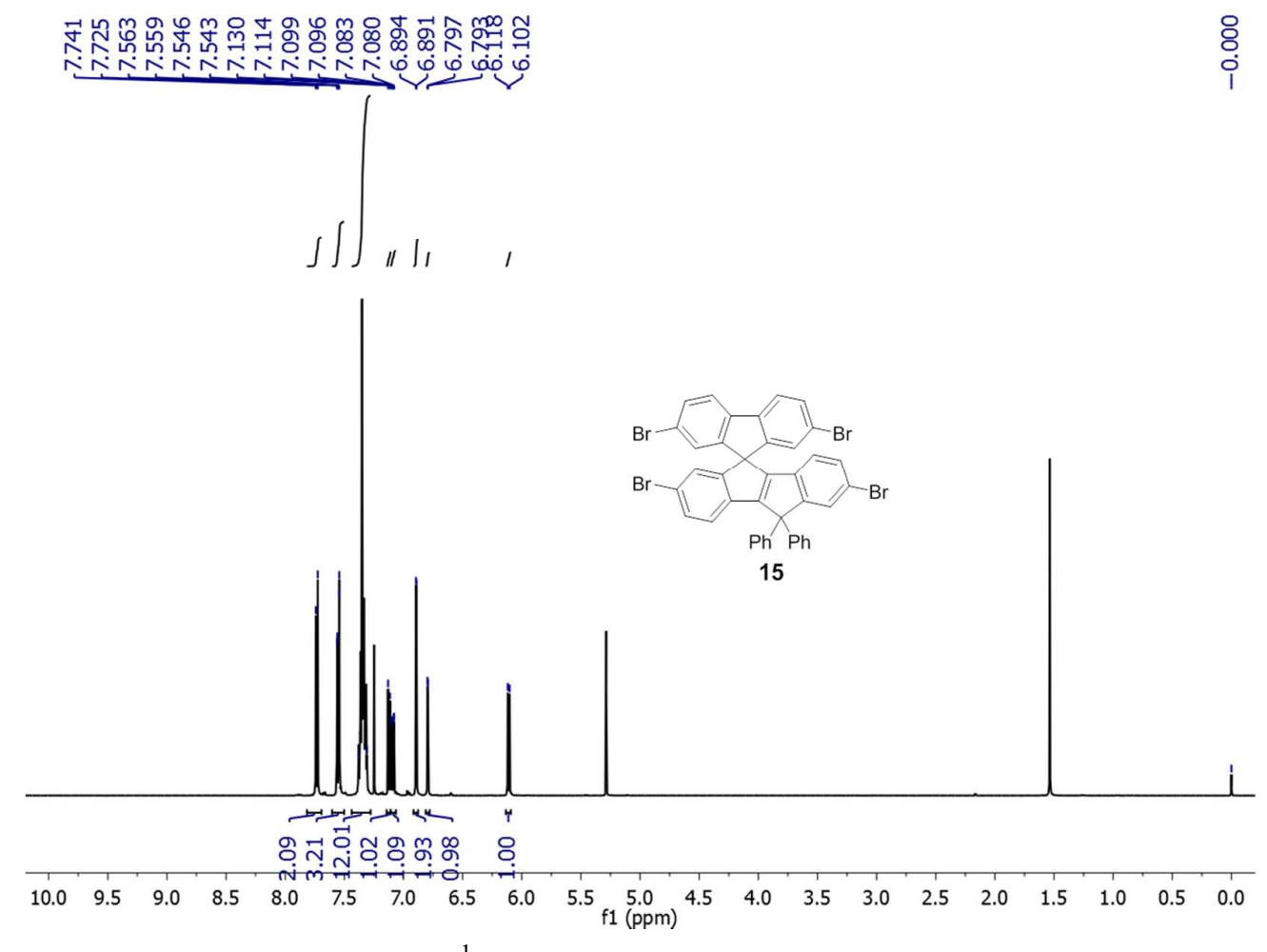

${ }^{1} \mathrm{H}$ NMR spectrum of $\mathbf{1 7}$
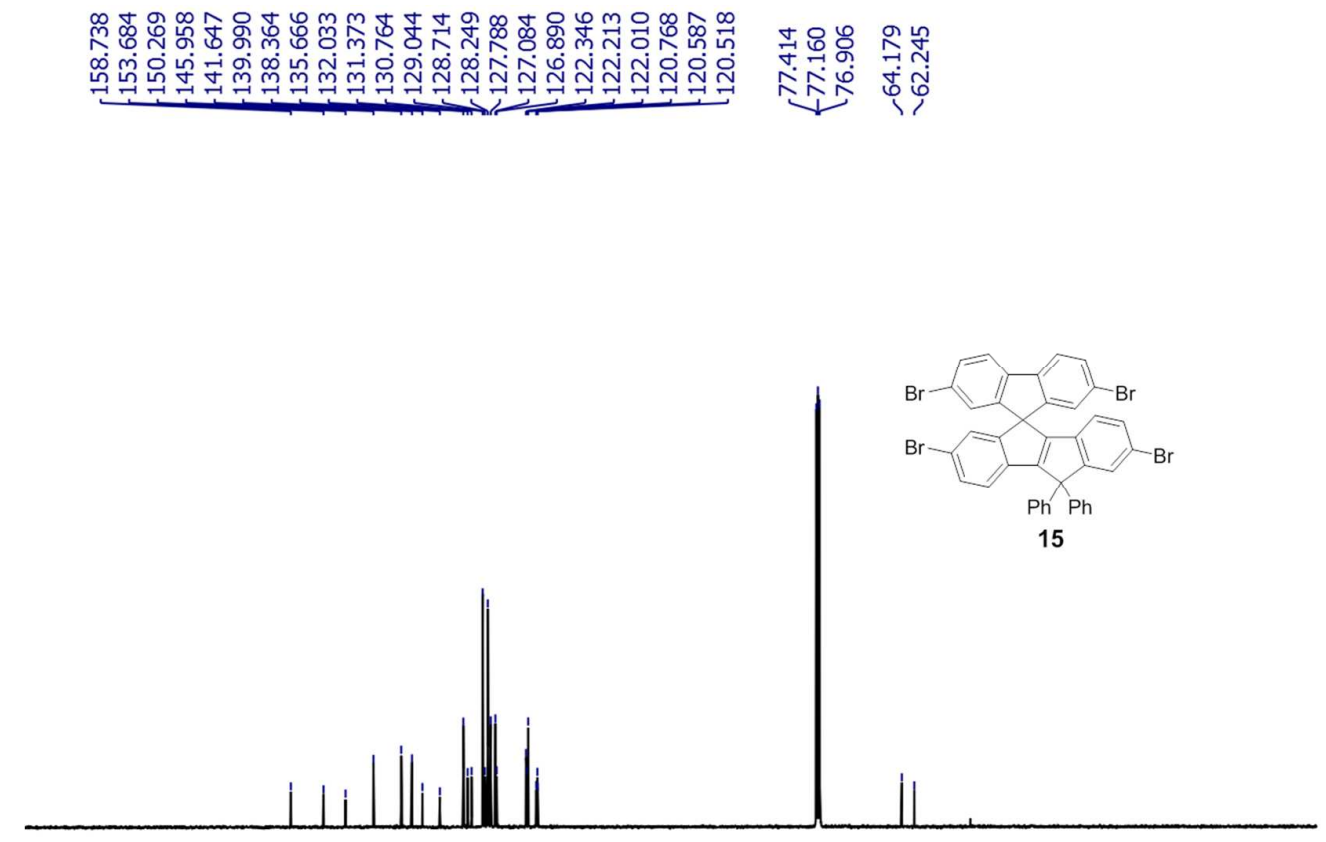

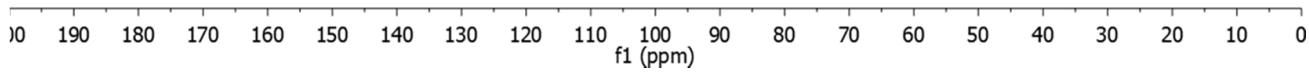
${ }^{13} \mathrm{C}$ NMR spectrum of 17 


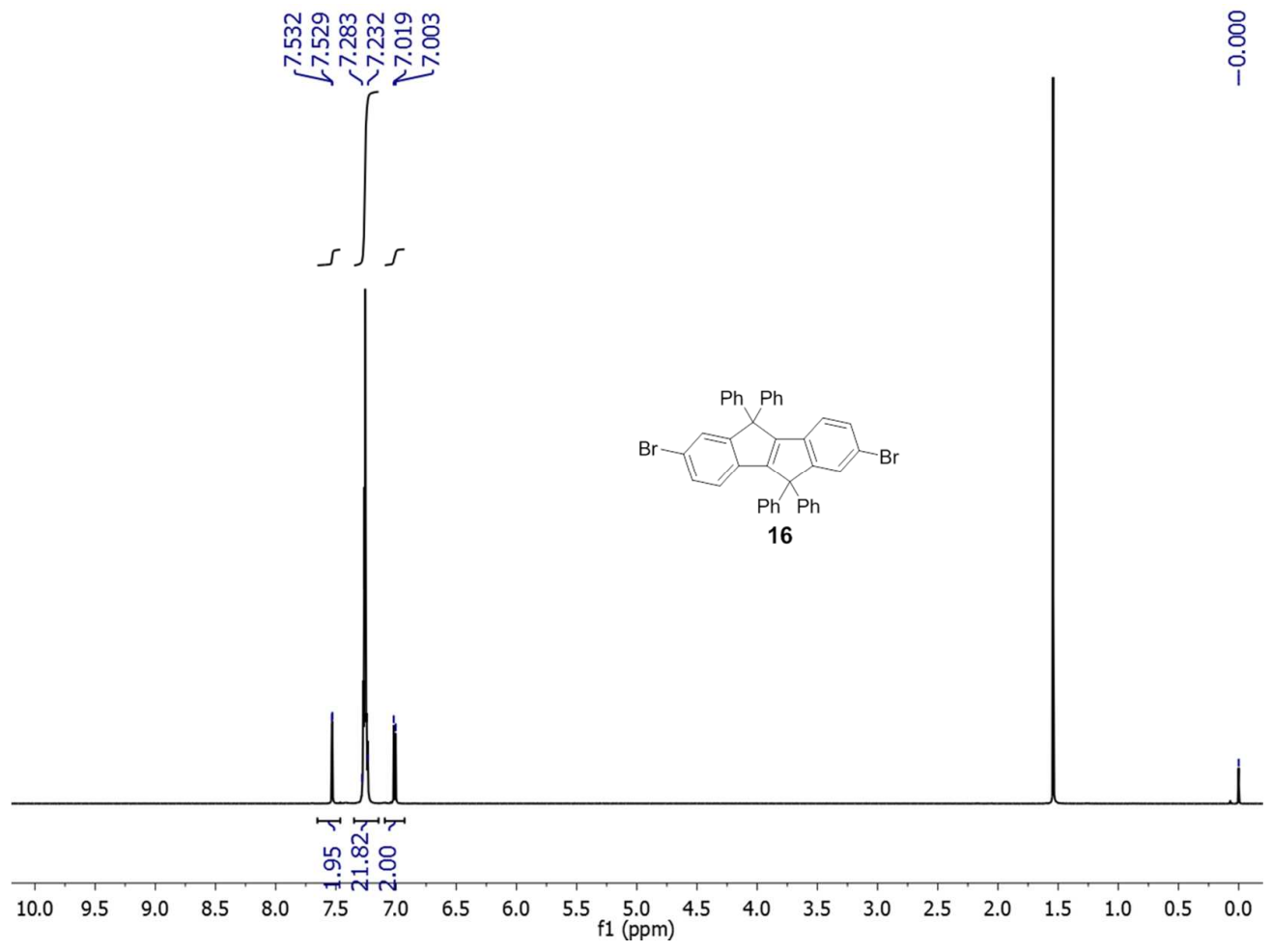

${ }^{1} \mathrm{H}$ NMR spectrum of $\mathbf{1 8}$

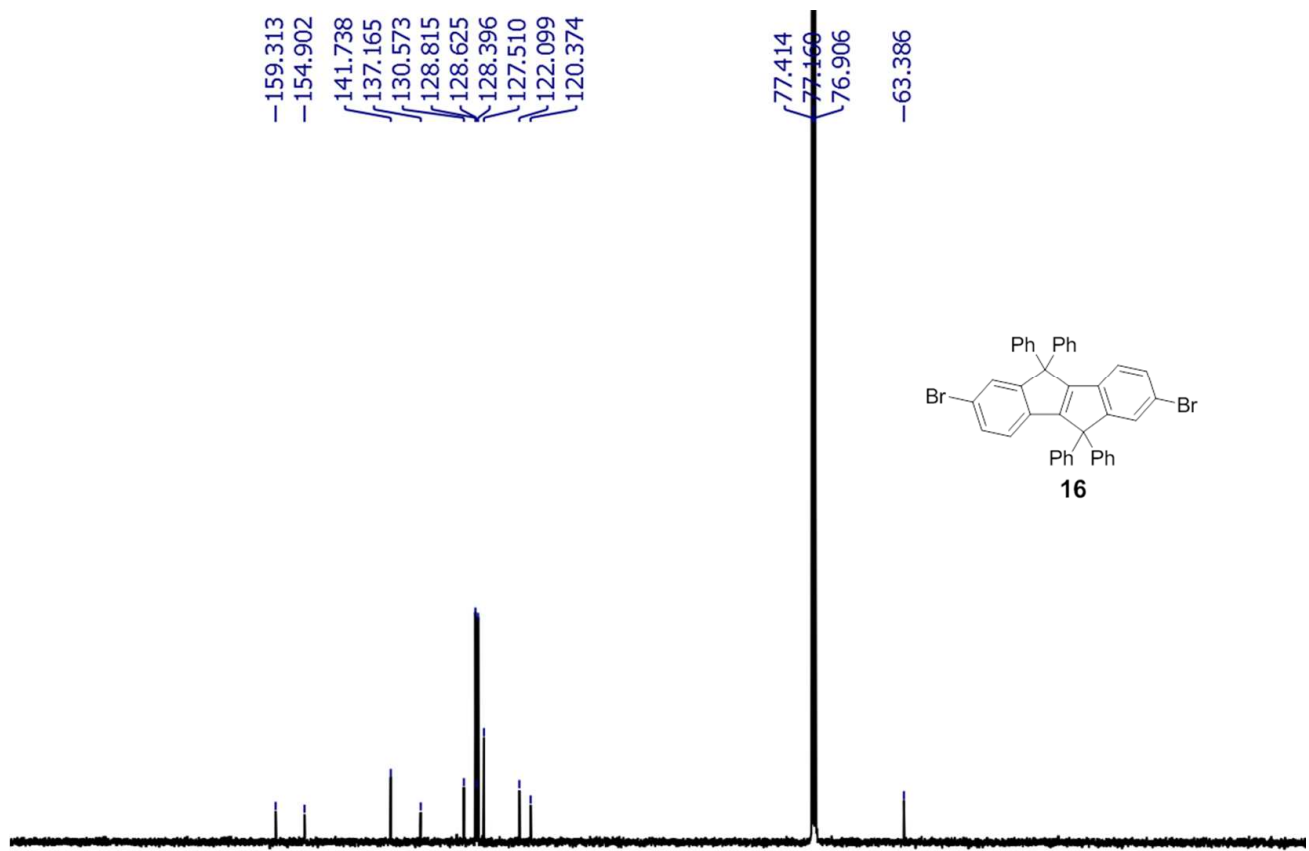

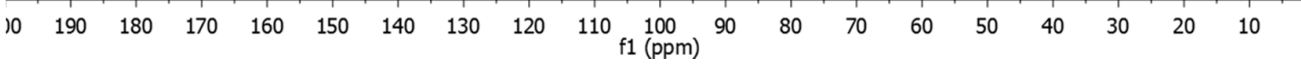

${ }^{13} \mathrm{C}$ NMR spectrum of $\mathbf{1 8}$ 

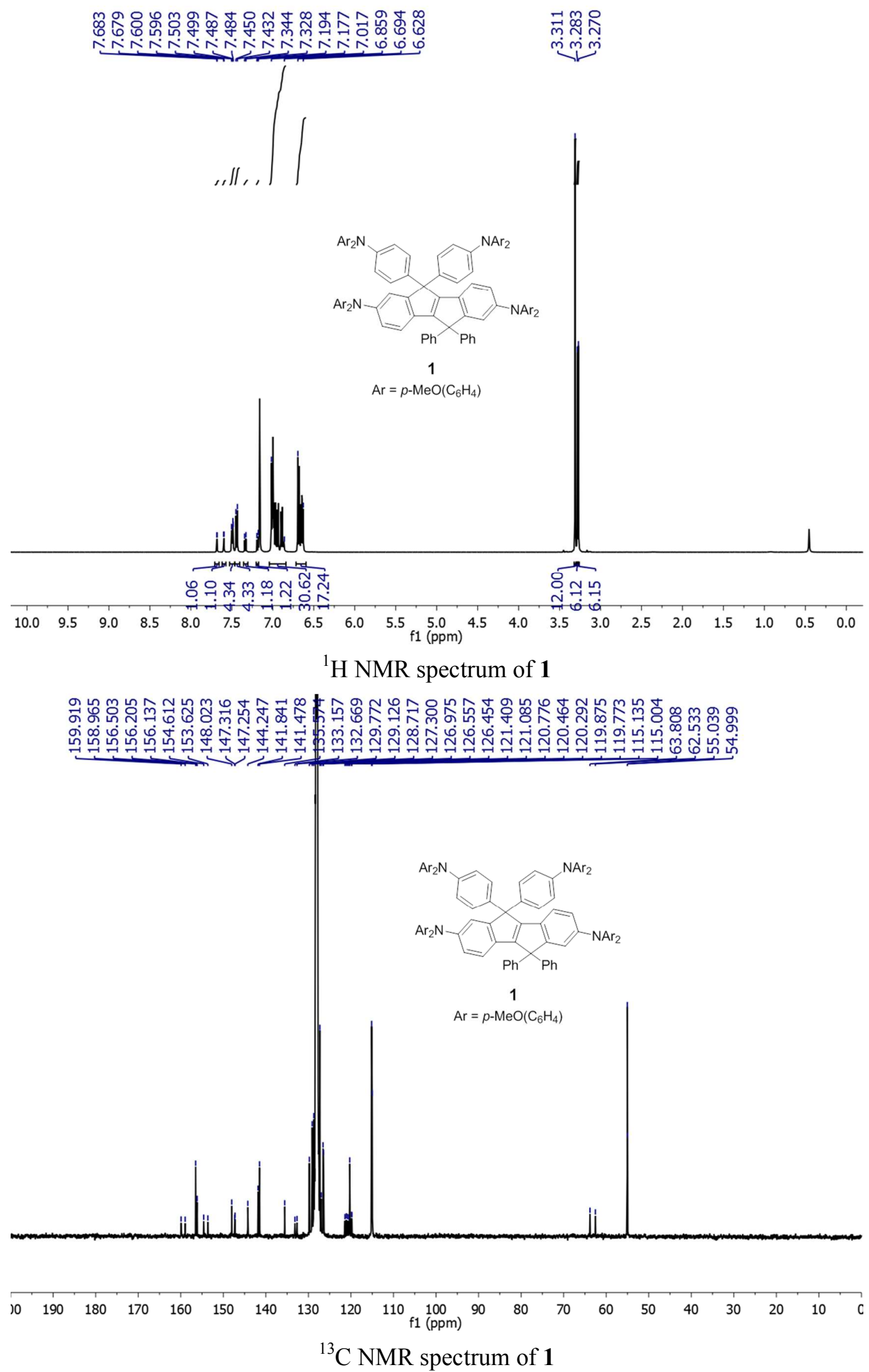

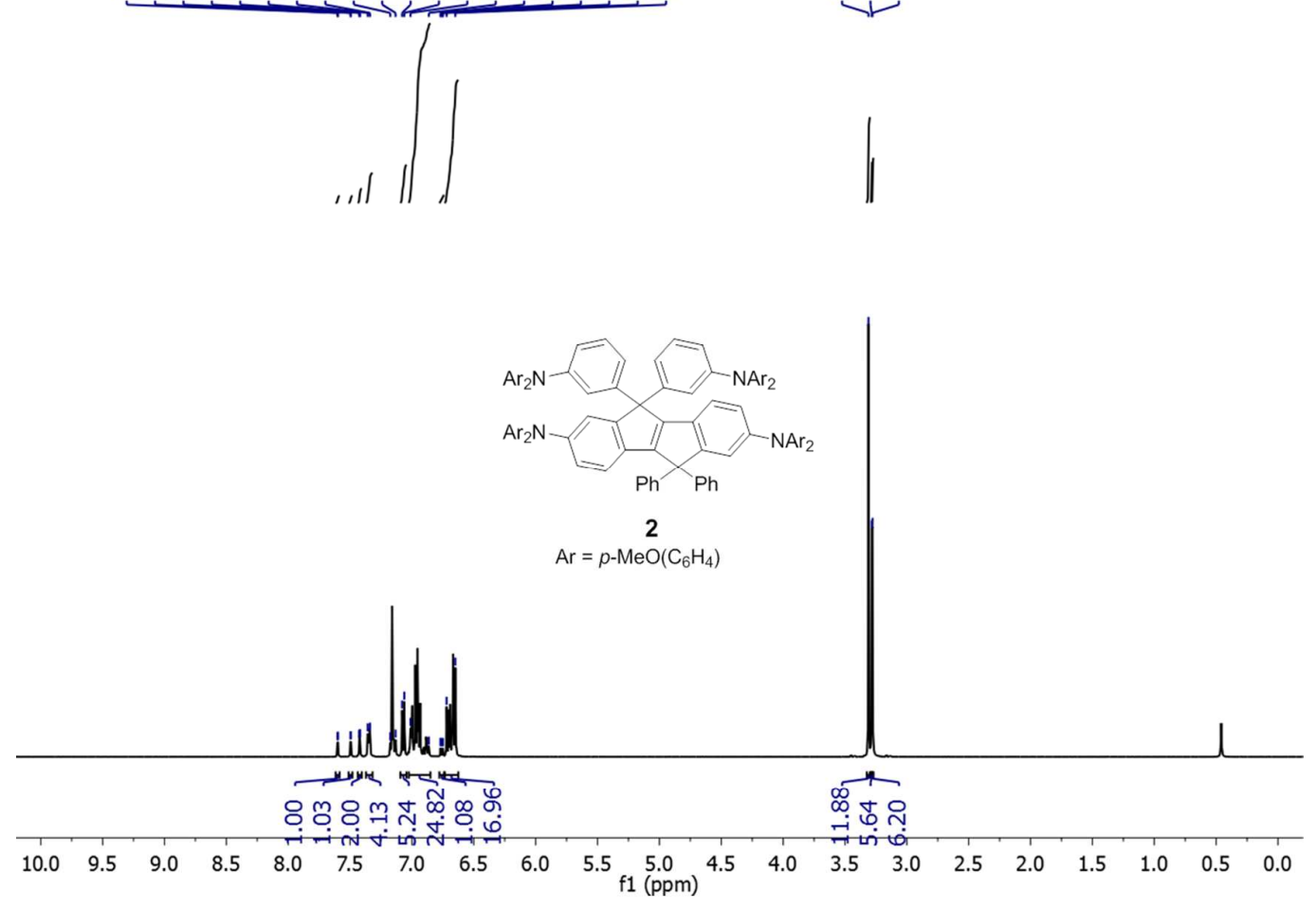

${ }^{1} \mathrm{H}$ NMR spectrum of 2

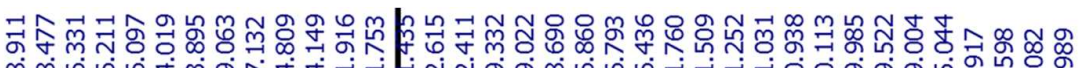

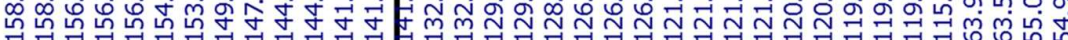

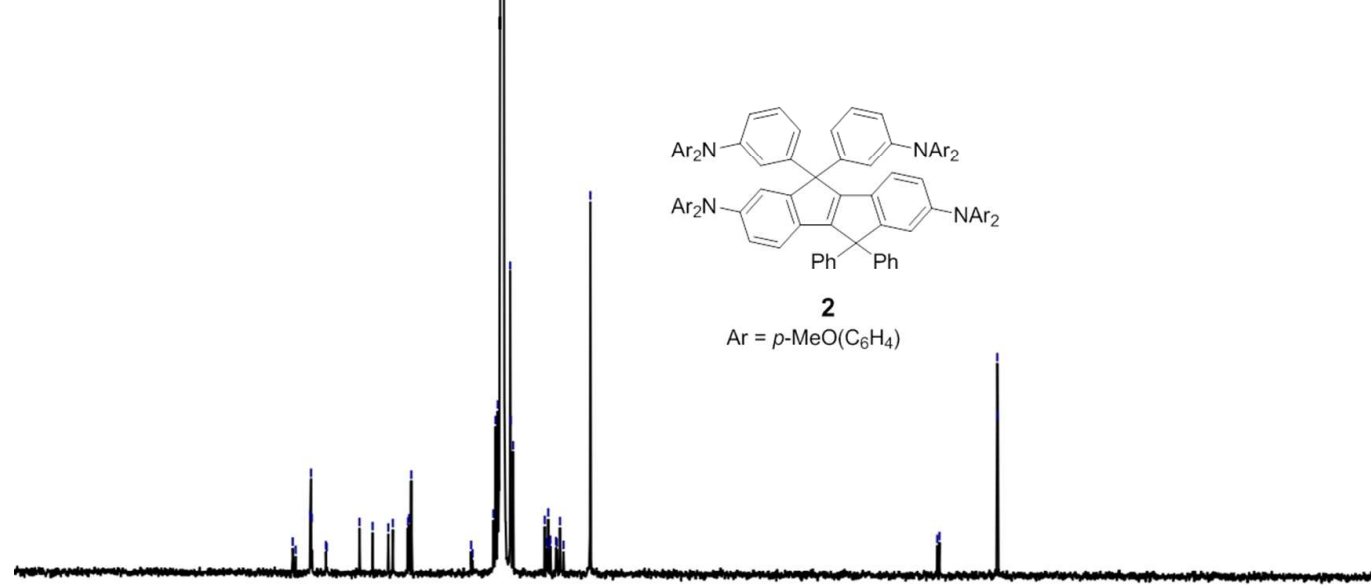

10 $190 \begin{array}{llllllllllllllllll}180 & 170 & 160 & 150 & 140 & 130 & 120 & 110 & \begin{array}{c}100 \\ \mathrm{f} 1(\mathrm{ppm})\end{array} & 90 & 80 & 70 & 60 & 50 & 40 & 30 & 20 & 10\end{array}$

${ }^{13} \mathrm{C}$ NMR spectrum of $\mathbf{2}$ 

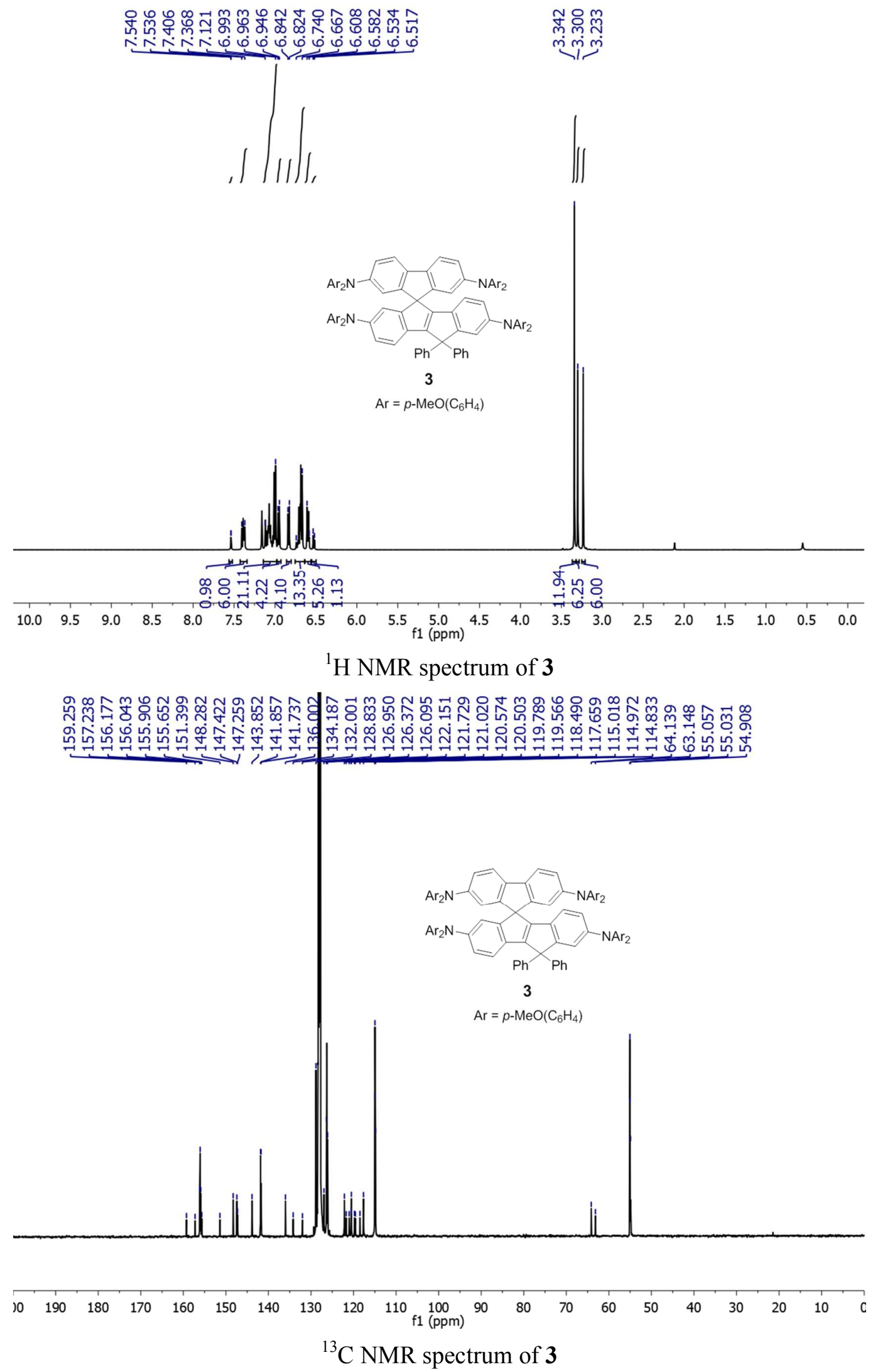


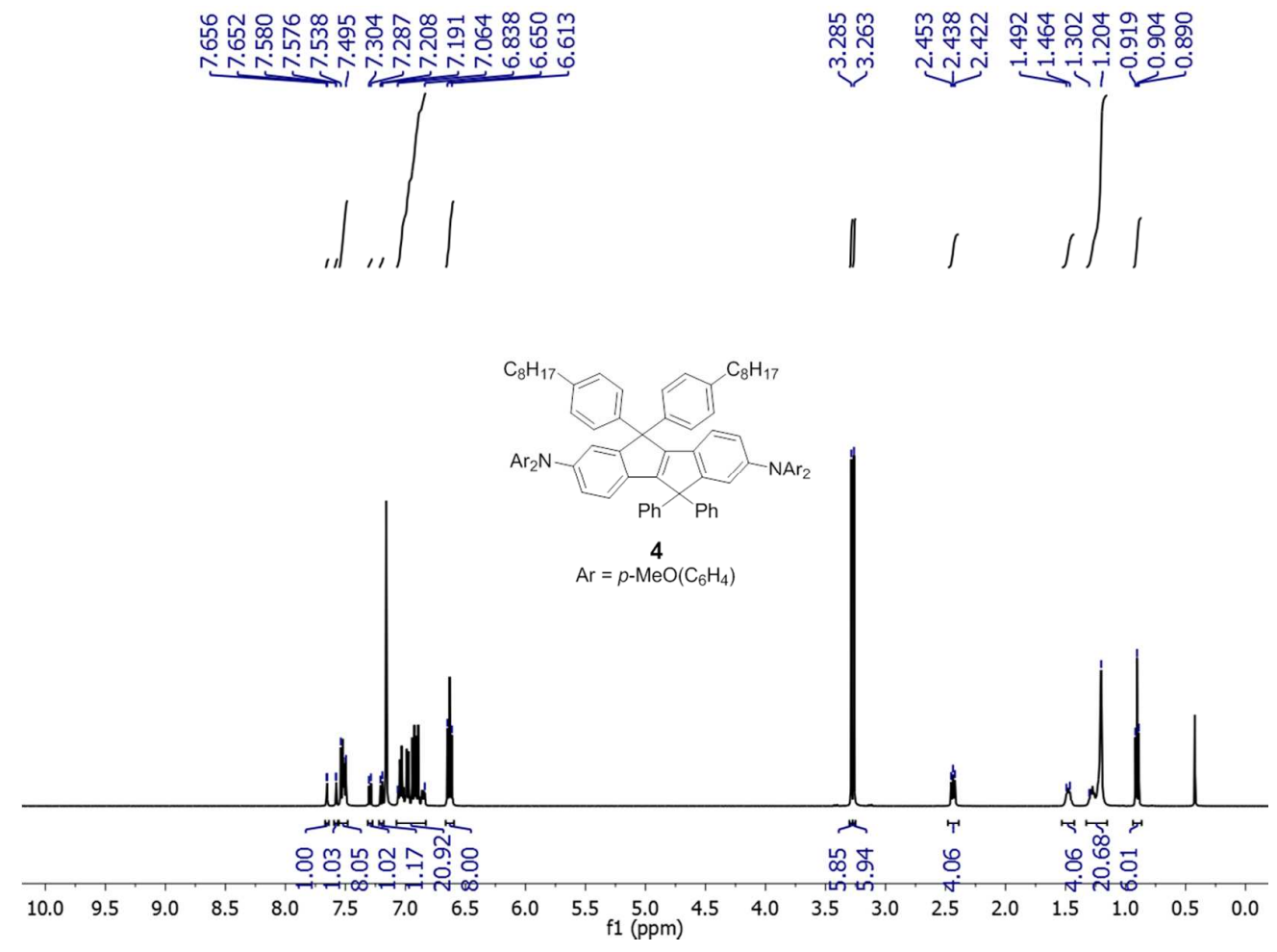

${ }^{1} \mathrm{H}$ NMR spectrum of 4

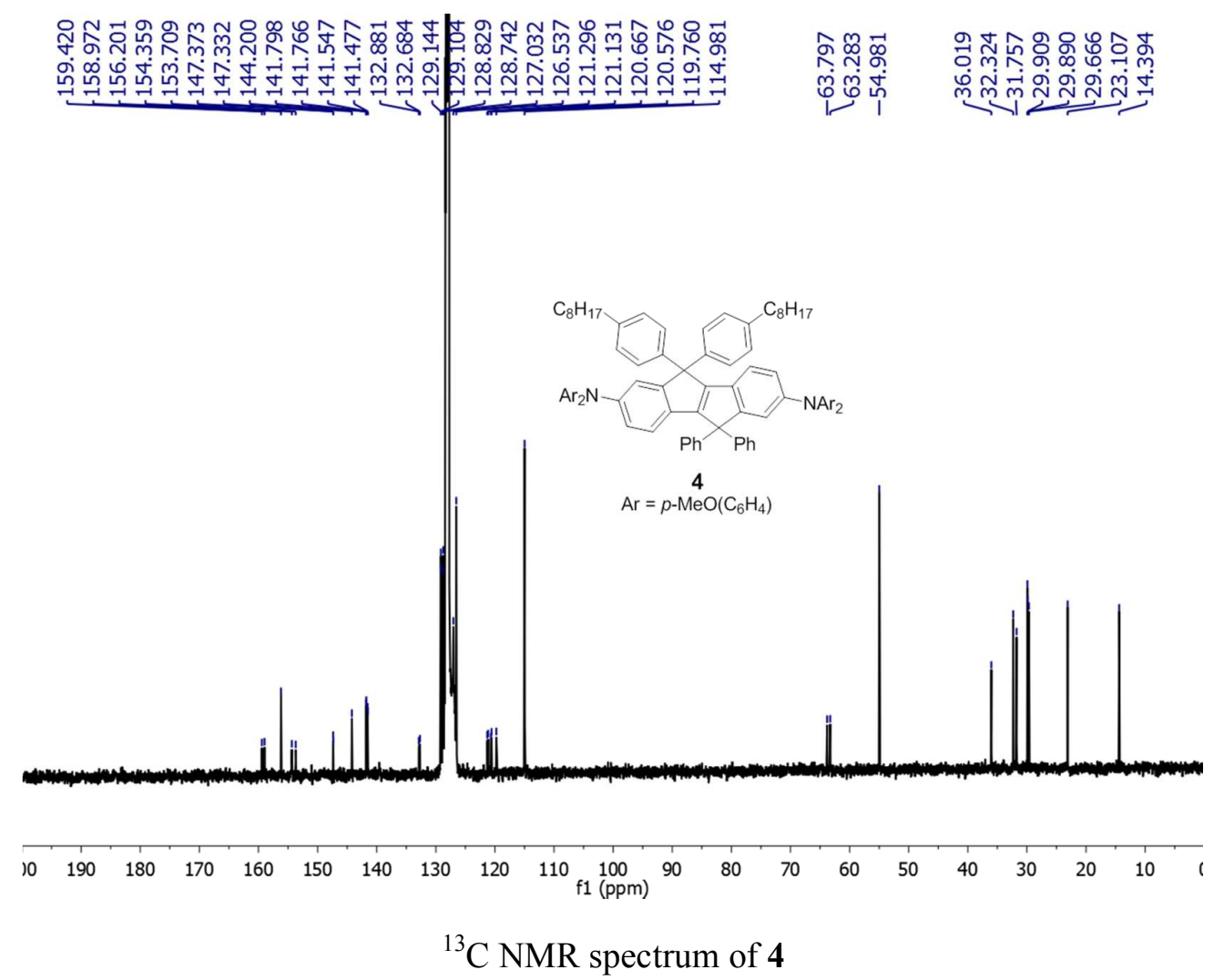



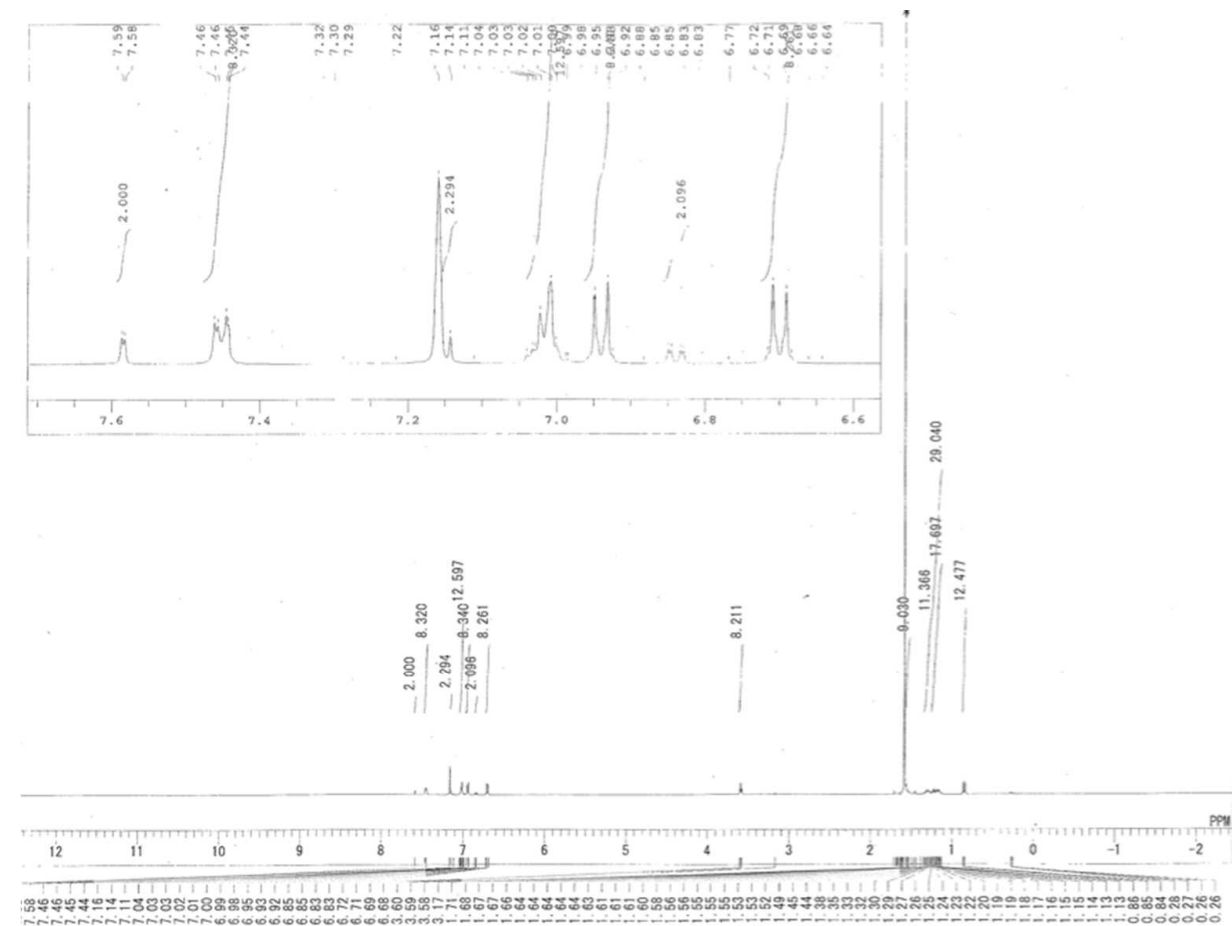

${ }^{1} \mathrm{H}$ NMR spectrum of $\mathbf{5}$

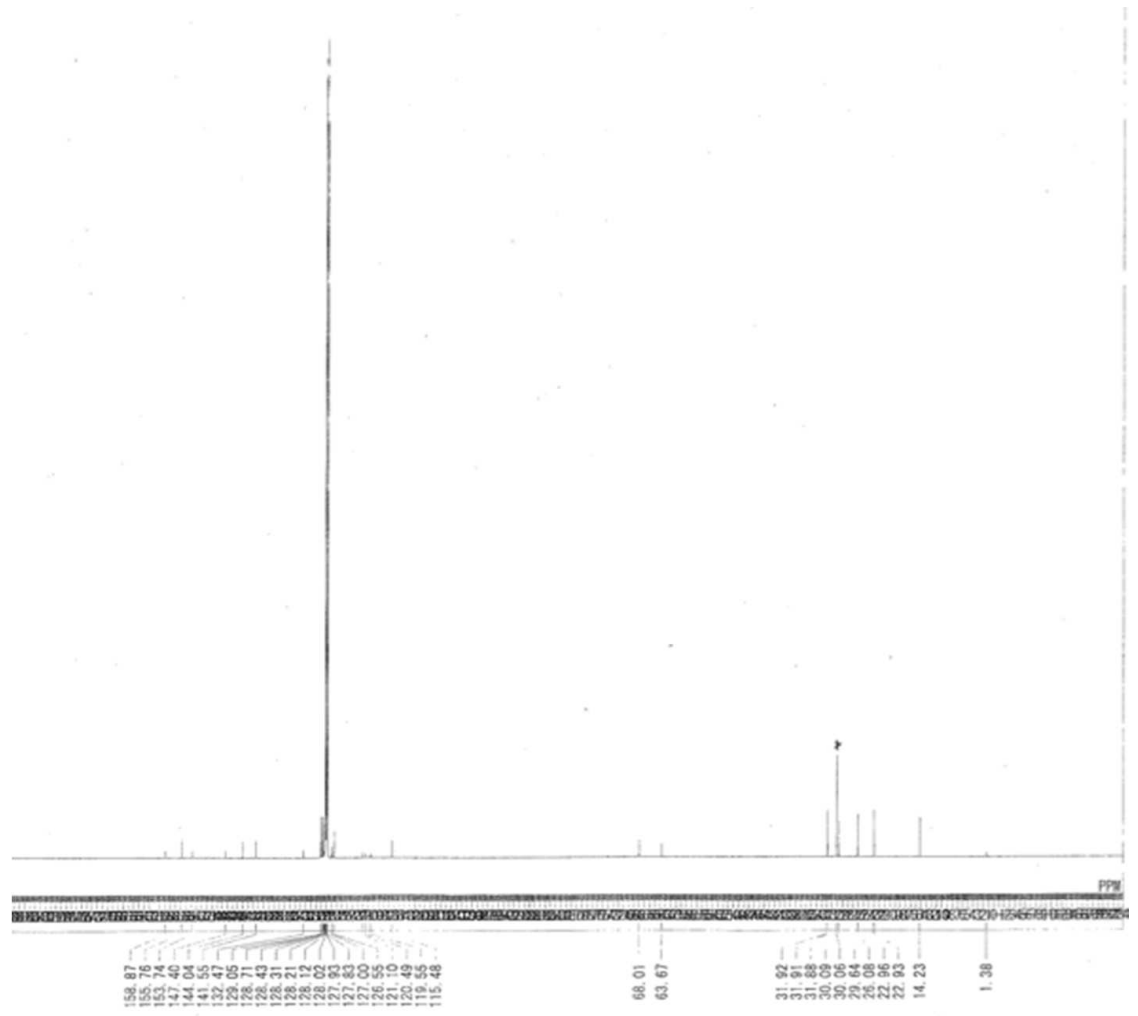

${ }^{13} \mathrm{C}$ NMR spectrum of 5 


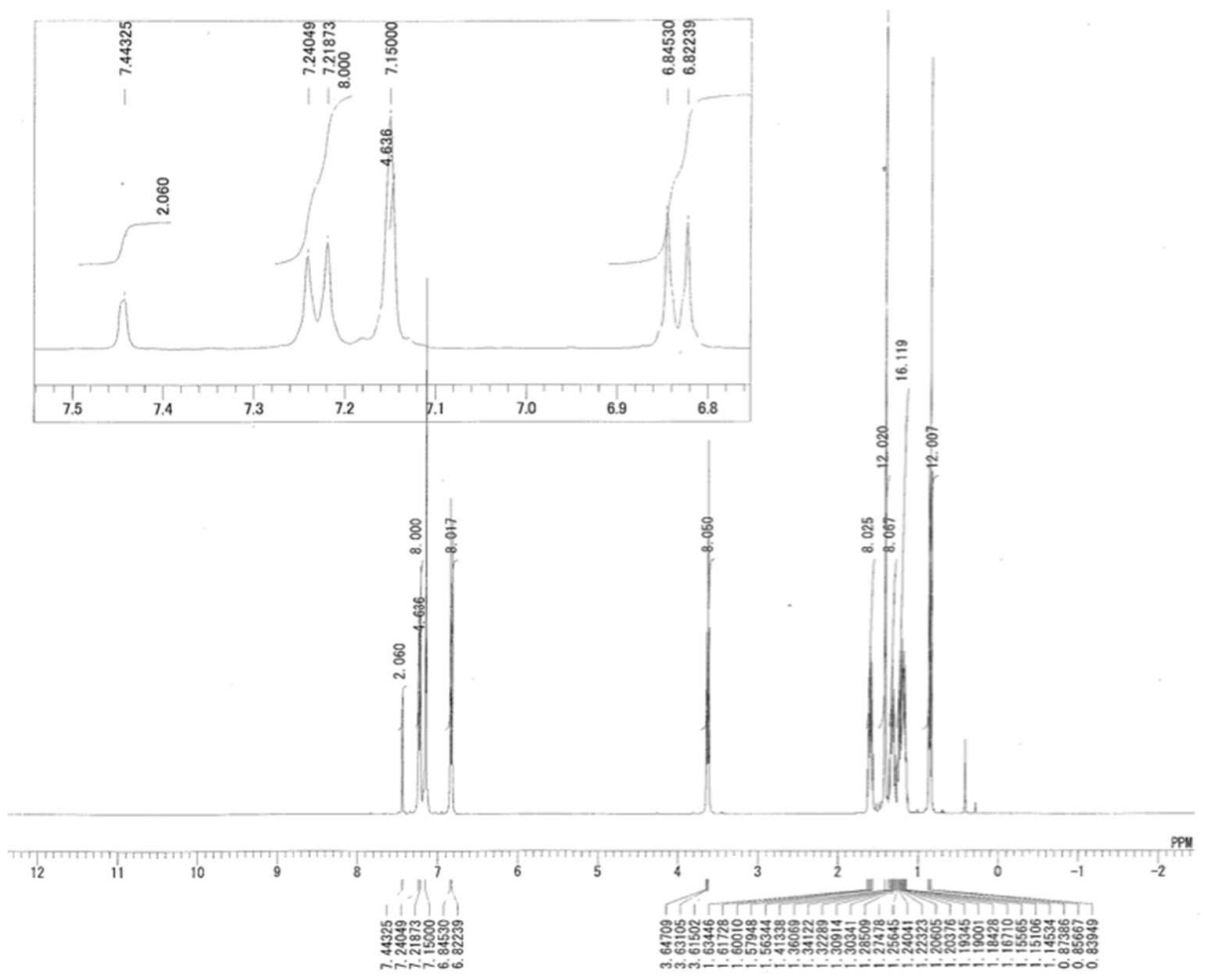

${ }^{1} \mathrm{H}$ NMR spectrum of 6

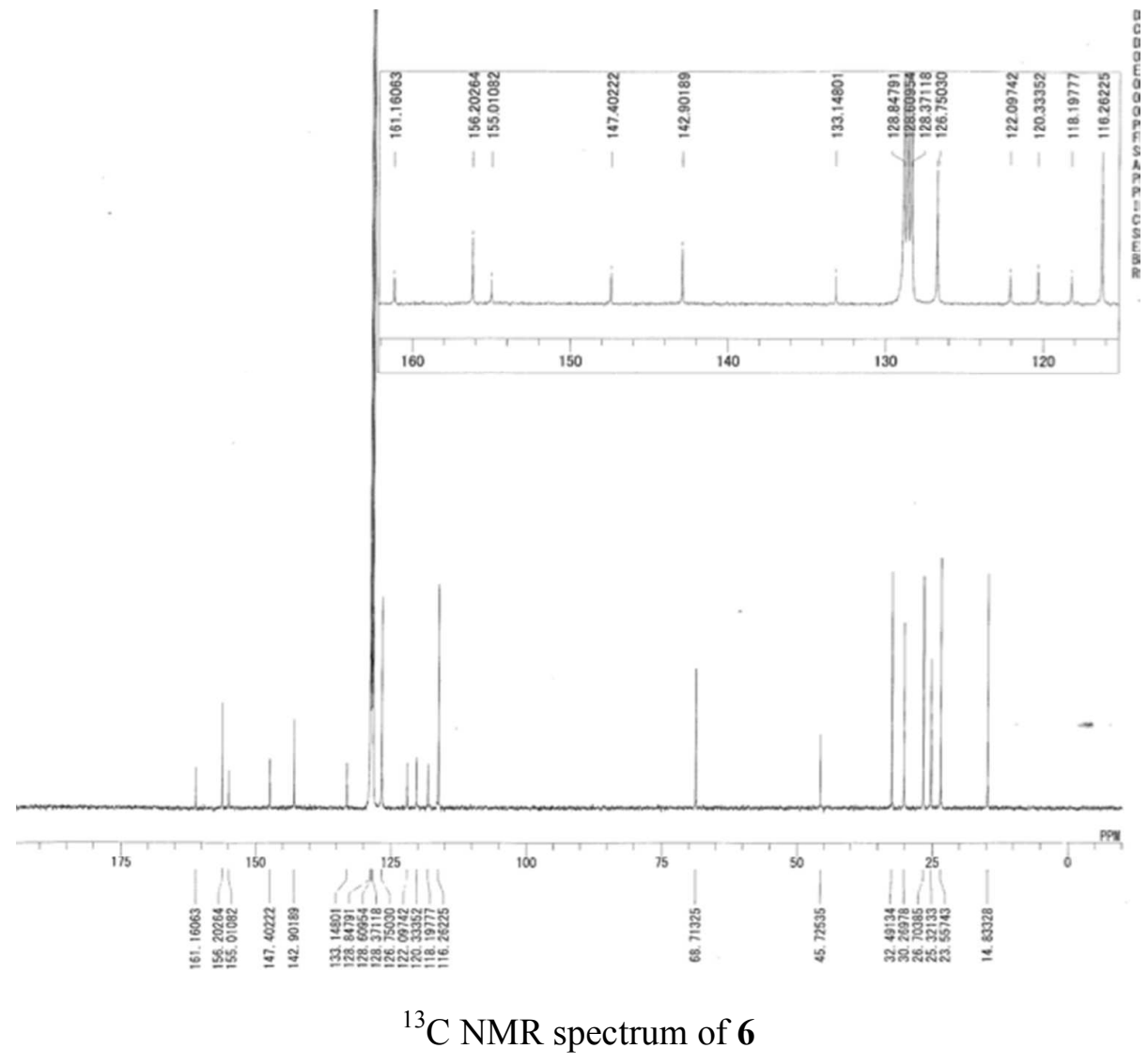




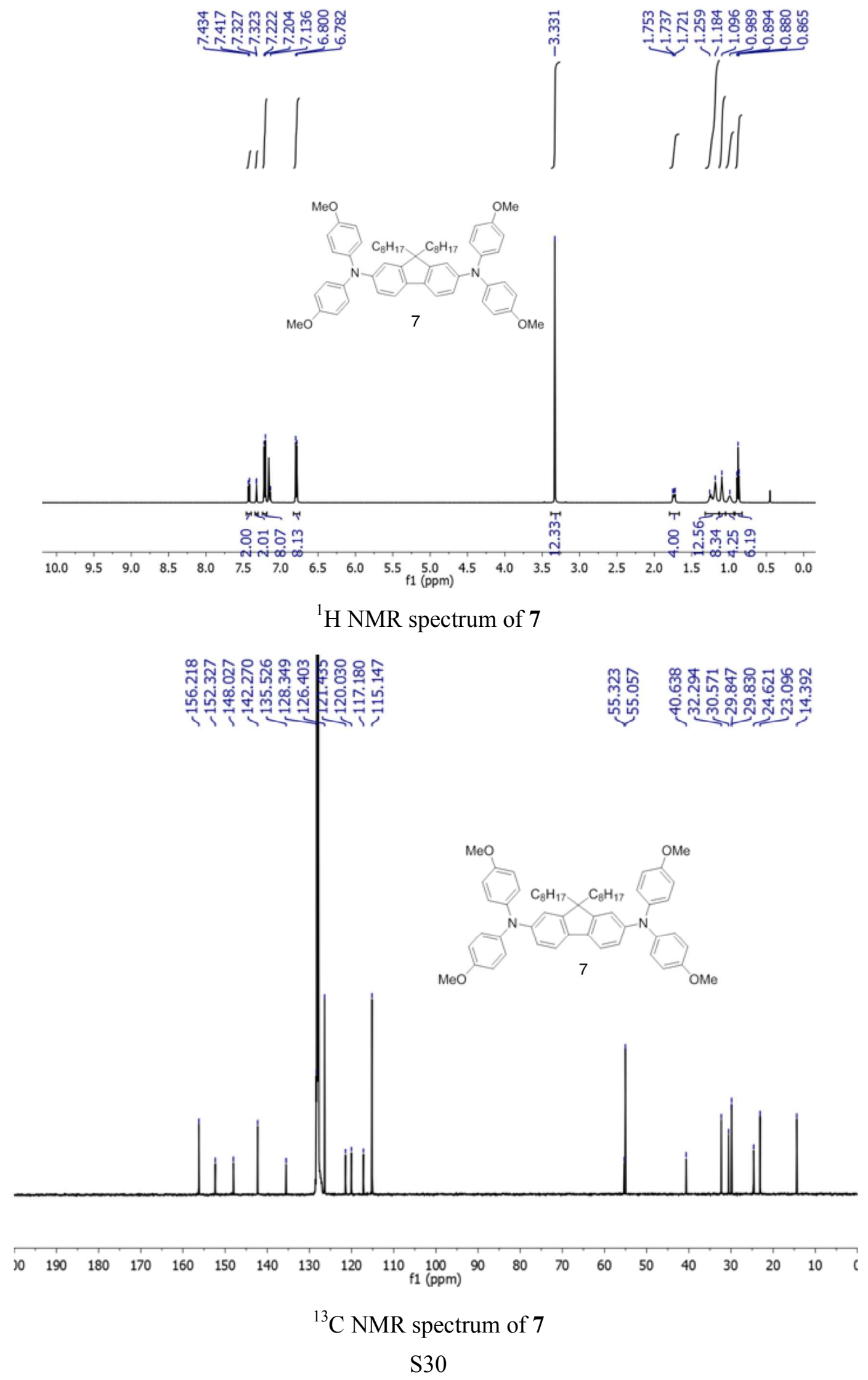

